\title{
An application of variational approach to a class of damped vibration problems with impulsive effects on time scales
}

Jianwen Zhou, Yanning Wang and Yongkun Li

$\overline{{ }^{*} \text { Correspondence: } y k \text { klie@ynu.edu.cn }}$ Department of Mathematics, Yunnan University, Kunming, Yunnan 650091, People's Republic of China

\begin{abstract}
In this paper, we present a new approach via variational methods and critical point theory to obtain the existence and multiplicity of solutions to a class of damped vibration problems with impulsive effects on time scales. By establishing a proper variational set, two existence results and two multiplicity results are obtained. Finally, one example is presented to illustrate the feasibility and effectiveness of our results.
\end{abstract}

Keywords: damped vibration problems; impulse; time scales

\section{Introduction}

Consider the damped vibration problem with impulsive effects on time scales

$$
\left\{\begin{array}{l}
u^{\Delta^{2}}(t)+B\left(u+u^{\sigma}\right)^{\Delta}(t)+A(\sigma(t)) u(\sigma(t)) \\
\quad+\nabla F(\sigma(t), u(\sigma(t)))=0, \quad \Delta \text {-a.e. } t \in[0, T]_{\mathbb{T}}^{\kappa}, \\
u(0)-u(T)=u^{\Delta}(0)-u^{\Delta}(T)=0, \\
\left(u^{i}\right)^{\Delta}\left(t_{j}^{+}\right)-\left(u^{i}\right)^{\Delta}\left(t_{j}^{-}\right)=I_{i j}\left(u^{i}\left(t_{j}\right)\right), \quad i=1,2, \ldots, N, j=1,2, \ldots, p,
\end{array}\right.
$$

where $t_{0}=0<t_{1}<t_{2}<\cdots<t_{p}<t_{p+1}=T, t_{j} \in[0, T]_{\mathbb{T}}(j=0,1,2, \ldots, p+1)$,

$$
\begin{aligned}
& \left(u^{i}\right)^{\Delta}\left(t_{j}^{+}\right)= \begin{cases}\lim _{t \rightarrow t_{j}^{+}}\left(u^{i}\right)^{\Delta}(t), & t \text { is right-dense; } \\
\left(u^{i}\right)^{\Delta}\left(\sigma\left(t_{j}\right)\right), & t \text { is right-scattered, }\end{cases} \\
& \left(u^{i}\right)^{\Delta}\left(t_{j}^{-}\right)= \begin{cases}\lim _{t \rightarrow t_{j}^{-}}\left(u^{i}\right)^{\Delta}(t), & t \text { is left-dense; } \\
\left(u^{i}\right)^{\Delta}\left(\rho\left(t_{j}\right)\right), & t \text { is left-scattered, }\end{cases}
\end{aligned}
$$

$u(t)=\left(u^{1}(t), u^{2}(t), \ldots, u^{N}(t)\right), B=\left[\bar{b}_{l m}\right]$ is an antisymmetry $N \times N$ constant matrix, $A(t)=$ $\left[\bar{a}_{l m}(t)\right]$ is a symmetric $N \times N$ matrix-valued function defined on $[0, T]_{\mathbb{T}}$ with $\bar{a}_{l m} \in$ $L^{\infty}([0, T], \mathbb{R})$, for all $l, m=1,2, \ldots, N, I_{i j}: \mathbb{R} \rightarrow \mathbb{R}(i=1,2, \ldots, N, j=1,2, \ldots, p)$ are continuous and $F:[0, T]_{\mathbb{T}} \times \mathbb{R}^{N} \rightarrow \mathbb{R}$ satisfies the following assumption:

(A) $F(t, x)$ is $\Delta$-measurable in $t$ for every $x \in \mathbb{R}^{N}$ and continuously differentiable in $x$ for $\Delta$-a.e. $t \in[0, T]_{\mathbb{T}}$ and there exist $a \in C\left(\mathbb{R}^{+}, \mathbb{R}^{+}\right), b^{\sigma} \in L^{1}\left(0, T ; \mathbb{R}^{+}\right)$such that

$$
|F(t, x)| \leq a(|x|) b(t), \quad|\nabla F(t, x)| \leq a(|x|) b(t),
$$

๑ 2015 Zhou et al.; licensee Springer. This is an Open Access article distributed under the terms of the Creative Commons Attribution License (http://creativecommons.org/licenses/by/4.0), which permits unrestricted use, distribution, and reproduction in any medium, provided the original work is properly credited. 
for all $x \in \mathbb{R}^{N}$ and $\Delta$-a.e. $t \in[0, T]_{\mathbb{T}}$, where $\nabla F(t, x)$ denotes the gradient of $F(t, x)$ in $x$.

For the sake of convenience, in the sequel, we denote $\Lambda_{1}=\{1,2, \ldots, N\}, \Lambda_{2}=\{1,2, \ldots, p\}$. Problem (1) covers the second-order Hamiltonian system with impulsive effects (when $\mathbb{T}=\mathbb{R})$

$$
\left\{\begin{array}{l}
\ddot{u}(t)+2 B \dot{u}(t)+A(t) u(t)+\nabla F(t, u(t))=0, \quad \text { a.e. } t \in[0, T] \\
u(0)-u(T)=\dot{u}(0)-\dot{u}(T)=0, \\
\dot{u}^{i}\left(t_{j}^{+}\right)-\dot{u}^{i}\left(t_{j}^{-}\right)=I_{i j}\left(u^{i}\left(t_{j}\right)\right), \quad i=1,2, \ldots, N, j=1,2, \ldots, p,
\end{array}\right.
$$

as well as the second-order discrete Hamiltonian system (when $\mathbb{T}=\mathbb{Z}, T \in \mathbb{N}, T \geq 2$ )

$$
\left\{\begin{array}{l}
\Delta^{2} u(t)+B \Delta(u(t)+u(t+1))+A(t+1) u(t+1) \\
\quad+\nabla F(t+1, u(t+1))=0, \quad \text { a.e. } t \in[0, T-1] \cap \mathbb{Z}, \\
u(0)-u(T)=0, \quad \Delta u(0)-\Delta u(T)=0, \\
\Delta u^{i}\left(t_{j}+1\right)-\Delta u^{i}\left(t_{j}-1\right)=I_{i j}\left(u^{i}\left(t_{j}\right)\right), \quad i=1,2, \ldots, N, j=1,2, \ldots, p .
\end{array}\right.
$$

When $\mathbb{T}=\mathbb{R}, I_{i j} \equiv 0, i \in \Lambda_{1}, j \in \Lambda_{2}, B$ and $A(t)$ are zero matrices, (1) is the Hamiltonian system

$$
\left\{\begin{array}{l}
\ddot{u}(t)+\nabla F(t, u(t))=0, \quad \text { a.e. } t \in[0, T] \\
u(0)-u(T)=\dot{u}(0)-\dot{u}(T)=0
\end{array}\right.
$$

Mawhin and Willem in [1] studied the periodic solutions of (2) and obtained a series of results. Equation (2) has also been investigated by several authors using various techniques and different conditions on the nonlinearities, such as the coercive type potential condition (see [2]) and the even type potential condition (see [3]).

When $\mathbb{T}=\mathbb{R}, I_{i j} \equiv 0, i \in \Lambda_{1}, j \in \Lambda_{2}, B=0$ and $A(t)$ is not a zero matrix, He and Wu in [4] researched the existence of solutions for (1) when $A(t)$ is negative-definite. Meng and Zhang in [5] got some sufficient conditions for the existence of solutions for (1) by using a minimax theorem. Wu et al. in [6] studied the periodic solutions for a class of damped vibration problems.

When $\mathbb{T}=\mathbb{R}, I_{i j} \equiv 0, i \in \Lambda_{1}, j \in \Lambda_{2}, B$ and $A(t)$ are not zero matrices, Li et al. in [7] researched the existence and multiplicity of solutions for (1) by variational methods and some critical point theorems.

When $I_{i j}(t) \not \equiv 0, i \in \Lambda_{1}, j \in \Lambda_{2}, B$ and $A(t)$ are not zero matrices, until now, it is unknown whether problem (1) has a variational structure or not.

In recent years, dynamic equations on time scales have been studied extensively in the literature (see [8-15]). The study of dynamic equations on a time scale is a new area of still fairly theoretical exploration in mathematics. Dynamic equations on time scales can build bridges between continuous and discrete mathematics. Hilger introduce the theory of time scales with the motivation of providing a unified approach to discrete and continuous analysis in [16]. In fact, the calculus on time scales can unify continuous and discrete analysis. There exist many other interesting time scales in the real world. The time scales calculus has a tremendous potential for applications in some mathematical problems and some mathematical models of real processes and phenomena studied in physics, chemical technology, population dynamics, biotechnology, economics, neural networks, and 
social sciences $[17,18]$. For example, it can model insect populations that are continuous in season (and may follow a difference scheme with variable step size), die out in winter, while their eggs are incubating or dormant, and then hatch in a new season, giving rise to a nonoverlapping population.

Besides, impulsive and periodic boundary value problems on time scales have been studied extensively in the literature. There have been many approaches to study periodic solutions of impulsive differential equations on time scales, such as method of lower and upper solutions, fixed-point theory, coincidence degree theory and so on. However, the study of solutions for impulsive differential equations on time scales using variational method has received considerably less attention. The variational method is, to the best of our knowledge, an effective approach to deal with nonlinear problems on time scales with some type of discontinuities such as impulses (see [19]).

Motivated by the above, we research the existence of variational construction for problem (1) in an appropriate space of functions in this paper. As applications, we study the existence and multiplicity of solutions for (1) by some critical point theorems. All these results are new even in both the differential equations case and the difference equations case.

\section{Preliminaries and statements}

In this section, we present some fundamental definitions and results from the calculus on time scales and Sobolev's spaces on time scales that will be required below.

We denote $[a, b]_{\mathbb{T}}^{\kappa^{2}}=\left([a, b]_{\mathbb{T}}^{\kappa}\right)^{\kappa}$, therefore $[a, b]_{\mathbb{T}}^{\kappa^{2}}=[a, b]_{\mathbb{T}}$ if $b$ is left-dense and $[a, b]_{\mathbb{T}}^{\kappa^{2}}=$ $[a, \rho(b)]_{\mathbb{T}}^{\kappa}$ if $b$ is left-scattered.

Definition 2.1 (Definition 2.3, [20]) Assume that $f: \mathbb{T} \rightarrow \mathbb{R}^{N}$ is a function, $f(t)=$ $\left(f^{1}(t), f^{2}(t), \ldots, f^{N}(t)\right)$ and let $t \in \mathbb{T}^{\kappa}$. Then we define $f^{\Delta}(t)=\left(f^{1^{\Delta}}(t), f^{2^{\Delta}}(t), \ldots, f^{N^{\Delta}}(t)\right)$ (provided it exists). We call $f^{\Delta}(t)$ the delta (or Hilger) derivative of $f$ at $t$. The function $f$ is delta (or Hilger) differentiable provided $f^{\Delta}(t)$ exists, for all $t \in \mathbb{T}^{\kappa}$. The function $f^{\Delta}: \mathbb{T}^{\kappa} \rightarrow \mathbb{R}^{N}$ is then called the delta derivative of $f$ on $\mathbb{T}^{\kappa}$.

Definition 2.2 (Definition 2.5, [20]) For a function $f: \mathbb{T} \rightarrow \mathbb{R}^{N}$ we shall refer to the second derivative $f^{\Delta^{2}}$ provided $f^{\Delta}$ is differentiable on $\mathbb{T}^{\kappa^{2}}=\left(\mathbb{T}^{\kappa}\right)^{\kappa}$ with derivative $f^{\Delta^{2}}=\left(f^{\Delta}\right)^{\Delta}$ : $\mathbb{T}^{\kappa^{2}} \rightarrow \mathbb{R}^{N}$

Definition 2.3 (Definition 2.7, [20]) Assume that $f: \mathbb{T} \rightarrow \mathbb{R}^{N}$ is a function, $f(t)=$ $\left(f^{1}(t), f^{2}(t), \ldots, f^{N}(t)\right)$ and let $A$ be a $\Delta$-measurable subset of $\mathbb{T}$. $f$ is integrable on $A$ if and only if $f^{i}(i=1,2, \ldots, N)$ are integrable on $A$, and $\int_{A} f(t) \Delta t=\left(\int_{A} f^{1}(t) \Delta t, \int_{A} f^{2}(t) \Delta t, \ldots\right.$, $\left.\int_{A} f^{N}(t) \Delta t\right)$.

Definition 2.4 ([20]) Let $B \subset \mathbb{T}$. $B$ is called a $\Delta$-null set if $\mu_{\Delta}(B)=0$. We say that a property $P$ holds $\Delta$-almost everywhere ( $\Delta$-a.e.) on $B$, or for $\Delta$-almost all ( $\Delta$-a.a.) $t \in B$ if there is a $\Delta$-null set $E_{0} \subset B$ such that $P$ holds, for all $t \in B \backslash E_{0}$.

For $p \in \mathbb{R}, p \geq 1$, we set the space

$$
L_{\Delta}^{p}\left([0, T)_{\mathbb{T}}, \mathbb{R}^{N}\right)=\left\{u:[0, T)_{\mathbb{T}} \rightarrow \mathbb{R}^{N}: \int_{[0, T)_{\mathbb{T}}}|f(t)|^{p} \Delta t<+\infty\right\}
$$


with the norm

$$
\|f\|_{L_{\Delta}^{p}}=\left(\int_{[0, T)_{\mathbb{T}}}|f(t)|^{p} \Delta t\right)^{\frac{1}{p}} .
$$

We have the following theorem.

Theorem 2.1 (Theorem 2.1, [20]) Let $p \in \mathbb{R}$ be such that $p \geq 1$. Then the space $L_{\Delta}^{p}\left([0, T)_{\mathbb{T}}\right.$, $\left.\mathbb{R}^{N}\right)$ is a Banach space together with the norm $\|\cdot\|_{L_{\Delta}^{p}}$. Moreover, $L_{\Delta}^{2}\left([a, b)_{\mathbb{T}}, \mathbb{R}^{N}\right)$ is a Hilbert space together with the inner product given for every $(f, g) \in L_{\Delta}^{p}\left([a, b)_{\mathbb{T}}, \mathbb{R}^{N}\right) \times$ $L_{\Delta}^{p}\left([a, b)_{\mathbb{T}}, \mathbb{R}^{N}\right)$ by

$$
\langle f, g\rangle_{L_{\Delta}^{2}}=\int_{[a, b)_{\mathbb{T}}}(f(t), g(t)) \Delta t
$$

where $(\cdot, \cdot)$ denotes the inner product in $\mathbb{R}^{N}$.

Definition 2.5 (Definition 2.11, [20]) A function $f:[a, b]_{\mathbb{T}} \rightarrow \mathbb{R}^{N}, f(t)=\left(f^{1}(t), f^{2}(t), \ldots\right.$, $\left.f^{N}(t)\right)$. We say that $f$ is absolutely continuous on $[a, b]_{\mathbb{T}}\left(\right.$ i.e. $f \in A C\left([a, b]_{\mathbb{T}}, \mathbb{R}^{N}\right)$ ), if, for every $\epsilon>0$, there exists $\delta>0$ such that if $\left\{\left[a_{k}, b_{k}\right)_{\mathbb{T}}\right\}_{k=1}^{n}$ is a finite pairwise disjoint family of subintervals of $[a, b]_{\mathbb{T}}$ satisfying $\sum_{k=1}^{n}\left(b_{k}-a_{k}\right)<\delta$, then $\sum_{k=1}^{n}\left|f\left(b_{k}\right)-f\left(a_{k}\right)\right|<\epsilon$.

Now, we recall the Sobolev space $W_{\Delta, T}^{1, p}\left([0, T]_{\mathbb{T}}, \mathbb{R}^{N}\right)$ on $[0, T]_{\mathbb{T}}$ defined in [20]. For the sake of convenience, in the sequel, we will let $u^{\sigma}(t)=u(\sigma(t))$.

Definition 2.6 (Definition 2.12, [20]) Let $p \in \mathbb{R}$ be such that $p>1$ and $u:[0, T]_{\mathbb{T}} \rightarrow \mathbb{R}^{N}$. We say that $u \in W_{\Delta, T}^{1, p}\left([0, T]_{\mathbb{T}}, \mathbb{R}^{N}\right)$ if and only if $u \in L_{\Delta}^{p}\left([0, T)_{\mathbb{T}}, \mathbb{R}^{N}\right)$ and there exists $g$ : $[0, T]_{\mathbb{T}}^{\kappa} \rightarrow \mathbb{R}^{N}$ such $g \in L_{\Delta}^{p}\left([0, T)_{\mathbb{T}}, \mathbb{R}^{N}\right)$ and

$$
\int_{[0, T)_{\mathbb{T}}}\left(u(t), \phi^{\Delta}(t)\right) \Delta t=-\int_{[0, T)_{\mathbb{T}}}\left(g(t), \phi^{\sigma}(t)\right) \Delta t, \quad \forall \phi \in C_{T, r d}^{1}\left([0, T]_{\mathbb{T}}, \mathbb{R}^{N}\right) .
$$

For $p \in \mathbb{R}, p>1$, we denote

$$
V_{\Delta, T}^{1, p}\left([0, T]_{\mathbb{T}}, \mathbb{R}^{N}\right)=\left\{x \in A C\left([0, T]_{\mathbb{T}}, \mathbb{R}^{N}\right): x^{\Delta} \in L_{\Delta}^{p}\left([0, T)_{\mathbb{T}}, \mathbb{R}^{N}\right), x(0)=x(T)\right\} .
$$

It follows from Remark 2.2 in [20] that

$$
V_{\Delta}^{1, p}\left([0, T]_{\mathbb{T}}, \mathbb{R}_{N}\right) \subset W_{\Delta}^{1, p}\left([0, T]_{\mathbb{T}}, \mathbb{R}^{N}\right)
$$

is true for every $p \in \mathbb{R}$ with $p>1$. These two sets are, as a class of functions, equivalent. It is the characterization of functions in $W_{\Delta, T}^{1, p}\left([0, T]_{\mathbb{T}}, \mathbb{R}^{N}\right)$ in terms of functions in $V_{\Delta, T}^{1, p}\left([0, T]_{\mathbb{T}}, \mathbb{R}^{N}\right)$ too. That is, we have the following theorem.

Theorem 2.2 (Theorem 2.5, [20]) Suppose that $u \in W_{\Delta, T}^{1, p}\left([0, T]_{\mathbb{T}}, \mathbb{R}^{N}\right)$ for some $p \in \mathbb{R}$ with $p>1$, and that (3) holds for $g \in L_{\Delta}^{p}\left([0, T)_{\mathbb{T}}, \mathbb{R}^{N}\right)$. Then, there exists a unique function $x \in$ $V_{\Delta, T}^{1, p}\left([0, T]_{\mathbb{T}}, \mathbb{R}^{N}\right)$ such that the equalities

$$
x=u, \quad x^{\Delta}=g, \quad \Delta \text {-a.e. on }[0, T)_{\mathbb{T}}
$$


are satisfied and

$$
\int_{[0, T)_{\mathbb{T}}} g(t) \Delta t=0
$$

By identifying $u \in W_{\Delta, T}^{1, p}\left([0, T]_{\mathbb{T}}, \mathbb{R}^{N}\right)$ with its absolutely continuous representative $x \in$ $V_{\Delta, T}^{1, p}\left([0, T]_{\mathbb{T}}, \mathbb{R}^{N}\right)$, for which (4) holds, the set $W_{\Delta, T}^{1, p}\left([0, T]_{\mathbb{T}}, \mathbb{R}^{N}\right)$ can be endowed with the structure of Banach space. That is, we have the following theorem.

Theorem 2.3 (Theorem 2.6, [18]) Assume $p \in \mathbb{R}$ and $p>1$. The set $W_{\Delta, T}^{1, p}\left([0, T]_{\mathbb{T}}, \mathbb{R}^{N}\right)$ is a Banach space together with the norm defined as

$$
\|u\|_{W_{\Delta, T}^{1, p}}=\left(\int_{[0, T)_{\mathbb{T}}}|u(t)|^{p} \Delta t+\int_{[0, T)_{\mathbb{T}}}\left|u^{\Delta}(t)\right|^{p} \Delta t\right)^{\frac{1}{p}}, \quad \forall u \in W_{\Delta, T}^{1, p}\left([0, T]_{\mathbb{T}}, \mathbb{R}^{N}\right) .
$$

Moreover, the set $H_{\Delta, T}^{1}=W_{\Delta, T}^{1,2}\left([0, T]_{\mathbb{T}}, \mathbb{R}^{N}\right)$ is a Hilbert space together with the inner product

$$
\langle u, v\rangle_{H_{\Delta, T}^{1}}=\int_{[0, T)_{\mathbb{T}}}(u(t), v(t)) \Delta t+\int_{[0, T)_{\mathbb{T}}}\left(u^{\Delta}(t), v^{\Delta}(t)\right) \Delta t, \quad \forall u, v \in H_{\Delta, T}^{1} .
$$

The Banach space $W_{\Delta, T}^{1, p}\left([0, T]_{\mathbb{T}}, \mathbb{R}^{N}\right)$ has some important properties.

Theorem 2.4 (Theorem 2.7, [20]) There exists $C_{1}>0$ such that the inequality

$$
\|u\|_{\infty} \leq C_{1}\|u\|_{H_{\Delta, T}^{1}}
$$

holds, for all $u \in H_{\Delta, T}^{1}$, where $\|u\|_{\infty}=\max _{t \in[0, T]_{\mathbb{T}}}|u(t)|$.

Moreover, if $\int_{[0, T)_{\mathbb{T}}} u(t) \Delta t=0$, then

$$
\|u\|_{\infty} \leq C_{1}\left\|u^{\Delta}\right\|_{L_{\Delta}^{2}}
$$

In the sequel, $\|\cdot\|$ denotes the norm $\|\cdot\|_{H_{\Delta, T}^{1}}$.

\section{Variational setting}

In this section, we recall some basic facts which will be used in the proofs of our main results. In order to apply the critical point theory, we make a variational structure. From this variational structure, we can reduce the problem of finding solutions of (1) to the one of seeking the critical points of a corresponding functional.

If $u \in H_{\Delta, T}^{1}$, by identifying $u \in H_{\Delta, T}^{1}$ with its absolutely continuous representative $x \in V_{\Delta, T}^{1,2}\left([0, T]_{\mathbb{T}}, \mathbb{R}^{N}\right)$ for which (4) holds, then $u$ is absolutely continuous and $\dot{u} \in$ $L^{2}\left([0, T)_{\mathbb{T}} ; \mathbb{R}^{N}\right)$. In this case, $\Delta u^{\Delta}\left(t^{+}\right)-u^{\Delta}\left(t^{-}\right)=0$ may not hold for some $t \in(0, T)_{\mathbb{T}}$. This leads to impulsive effects.

Take $v \in H_{\Delta, T}^{1}$ and multiply the two sides of the equality

$$
u^{\Delta^{2}}(t)+B\left(u+u^{\sigma}\right)^{\Delta}(t)+A(\sigma(t)) u(\sigma(t))+\nabla F(\sigma(t), u(\sigma(t)))=0
$$


by $v^{\sigma}$ and integrate on $[0, T)_{\mathbb{T}}$, we have

$$
\int_{[0, T)_{\mathbb{T}}}\left[u^{\Delta^{2}}(t)+B\left(u+u^{\sigma}\right)^{\Delta}(t)+A(\sigma(t)) u(\sigma(t))+\nabla F(\sigma(t), u(\sigma(t)))\right] v^{\sigma}(t) \Delta t=0 .
$$

Moreover, combining with $u^{\Delta}(0)-u^{\Delta}(T)=0$, one has

$$
\begin{aligned}
& \int_{[0, T)_{\mathbb{T}}}\left(u^{\Delta^{2}}(t), v^{\sigma}(t)\right) \Delta t \\
& \quad=\sum_{j=0}^{p} \int_{\left[t_{j}, t_{j+1}\right)_{\mathbb{T}}}\left(u^{\Delta^{2}}(t), v^{\sigma}(t)\right) \Delta t \\
& \quad=\sum_{j=0}^{p}\left[\left(u^{\Delta}\left(t_{j+1}^{-}\right), v\left(t_{j+1}^{-}\right)\right)-\left(u^{\Delta}\left(t_{j}^{+}\right), v\left(t_{j}^{+}\right)\right)-\int_{\left[t_{j}, t_{j+1}\right)}\left(u^{\Delta}(t), v^{\Delta}(t)\right) \Delta t\right] \\
& =\sum_{j=0}^{p}\left[\sum_{i=1}^{N}\left(\left(u^{i}\right)^{\Delta}\left(t_{j+1}^{-}\right) v^{i}\left(t_{j+1}^{-}\right)-\left(u^{i}\right)^{\Delta}\left(t_{j}^{+}\right) v^{i}\left(t_{j}^{+}\right)\right)-\int_{\left[t_{j}, t_{j+1}\right)}\left(u^{\Delta}(t), v^{\Delta}(t)\right) \Delta t\right] \\
& =u^{\Delta}(T) v(T)-u^{\Delta}(0) v(0)-\sum_{j=1}^{p} \sum_{i=1}^{N} I_{i j}\left(u^{i}\left(t_{j}\right)\right) v^{i}\left(t_{j}\right)-\int_{[0, T)_{\mathbb{T}}}\left(u^{\Delta}(t), v^{\Delta}(t)\right) \Delta t \\
& =-\sum_{j=1}^{p} \sum_{i=1}^{N} I_{i j}\left(u^{i}\left(t_{j}\right)\right) v^{i}\left(t_{j}\right)-\int_{[0, T)_{\mathbb{T}}}\left(u^{\Delta}(t), v^{\Delta}(t)\right) \Delta t
\end{aligned}
$$

and

$$
\begin{aligned}
\int_{[0, T)_{\mathbb{T}}}\left(B u^{\Delta}(t)+B\left(u^{\sigma}\right)^{\Delta}(t), v^{\sigma}(t)\right) \Delta t \\
\quad=\int_{[0, T)_{\mathbb{T}}}\left(B u^{\Delta}(t), v^{\sigma}(t)\right) \Delta t-\int_{[0, T)_{\mathbb{T}}}\left(B u^{\sigma}(t), v^{\Delta}(t)\right) \Delta t \\
\quad=\int_{[0, T)_{\mathbb{T}}}\left(B u^{\Delta}(t), v^{\sigma}(t)\right) \Delta t+\int_{[0, T)_{\mathbb{T}}}\left(B u^{\Delta}(t), v(t)\right) \Delta t .
\end{aligned}
$$

Considering the above, we introduce the following concept solution for problem (1).

Definition 3.1 We say that a function $u \in H_{\Delta, T}^{1}$ is a weak solution of problem (1) if the identity

$$
\begin{aligned}
& \int_{[0, T)_{\mathbb{T}}}\left(u^{\Delta}(t), v^{\Delta}(t)\right) \Delta t+\sum_{j=1}^{p} \sum_{i=1}^{N} I_{i j}\left(u^{i}\left(t_{j}\right)\right) v^{i}\left(t_{j}\right) \\
& =\int_{[0, T)_{\mathbb{T}}}\left(A^{\sigma}(t) u^{\sigma}(t)+\nabla F\left(\sigma(t), u^{\sigma}(t)\right), v^{\sigma}(t)\right) \Delta t \\
& \quad+\int_{[0, T)_{\mathbb{T}}}\left(B u^{\Delta}(t), v^{\sigma}(t)\right) \Delta t+\int_{[0, T)_{\mathbb{T}}}\left(B u^{\Delta}(t), v(t)\right) \Delta t
\end{aligned}
$$

holds for any $v \in H_{\Delta, T}^{1}$. 
Consider the functional $\varphi: H_{\Delta, T}^{1} \rightarrow \mathbb{R}$ defined by

$$
\begin{aligned}
\varphi(u)= & \frac{1}{2} \int_{[0, T)_{\mathbb{T}}}\left|u^{\Delta}(t)\right|^{2} \Delta t+\sum_{j=1}^{p} \sum_{i=1}^{N} \int_{0}^{u^{i}\left(t_{j}\right)} I_{i j}(t) \mathrm{d} t \\
& +\int_{[0, T)_{\mathbb{T}}}\left(B u^{\sigma}(t), u^{\Delta}(t)\right) \Delta t-\frac{1}{2} \int_{[0, T)_{\mathbb{T}}}\left(A^{\sigma}(t) u^{\sigma}(t), u^{\sigma}(t)\right) \Delta t+J(u) \\
= & \psi(u)+\phi(u),
\end{aligned}
$$

where

$$
\begin{aligned}
J(u)= & -\int_{[0, T)_{\mathbb{T}}} F\left(\sigma(t), u^{\sigma}(t)\right) \Delta t, \\
\psi(u)= & \frac{1}{2} \int_{[0, T)_{\mathbb{T}}}\left|u^{\Delta}(t)\right|^{2} \Delta t+\int_{[0, T)_{\mathbb{T}}}\left(B u^{\sigma}(t), u^{\Delta}(t)\right) \Delta t \\
& -\frac{1}{2} \int_{[0, T)_{\mathbb{T}}}\left(A^{\sigma}(t) u^{\sigma}(t), u^{\sigma}(t)\right) \Delta t+J(u), \\
\phi(u)= & \sum_{j=1}^{p} \sum_{i=1}^{N} \int_{0}^{u^{i}\left(t_{j}\right)} I_{i j}(t) \mathrm{d} t .
\end{aligned}
$$

Lemma 3.1 The functional $\varphi$ is continuously differentiable on $H_{\Delta, T}^{1}$ and

$$
\begin{aligned}
\left\langle\varphi^{\prime}(u), v\right\rangle= & \int_{[0, T)_{\mathbb{T}}}\left(u^{\Delta}(t), v^{\Delta}(t)\right) \Delta t+\sum_{j=1}^{p} \sum_{i=1}^{N} I_{i j}\left(u^{i}\left(t_{j}\right)\right) v^{i}\left(t_{j}\right) \\
& -\int_{[0, T)_{\mathbb{T}}}\left(A^{\sigma}(t) u^{\sigma}(t)+\nabla F\left(\sigma(t), u^{\sigma}(t)\right), v^{\sigma}(t)\right) \mathrm{d} t \\
& -\int_{[0, T)_{\mathbb{T}}}\left(B u^{\Delta}(t), v^{\sigma}(t)\right) \Delta t-\int_{[0, T)_{\mathbb{T}}}\left(B u^{\Delta}(t), v(t)\right) \Delta t .
\end{aligned}
$$

Proof Set $L(t, x, y)=\frac{1}{2}|y|^{2}+\frac{1}{2}(B x, y)-\frac{1}{2}(A(t) x, x)-F(t, x)$, for all $x, y \in R^{N}$ and $t \in[0, T)_{\mathbb{T}}$. Then $L(t, x, y)$ satisfies all assumptions of Theorem 2.9 in [18]. Hence, by Theorem 2.9 in [18], we know that the functional $\psi$ is continuously differentiable on $H_{\Delta, T}^{1}$ and

$$
\begin{aligned}
\left\langle\varphi^{\prime}(u), v\right\rangle= & \int_{[0, T)_{\mathbb{T}}}\left(u^{\Delta}(t), v^{\Delta}(t)\right) \Delta t+\sum_{j=1}^{p} \sum_{i=1}^{N} I_{i j}\left(u^{i}\left(t_{j}\right)\right) v^{i}\left(t_{j}\right) \\
& -\int_{[0, T)_{\mathbb{T}}}\left(A^{\sigma}(t) u^{\sigma}(t)+\nabla F\left(\sigma(t), u^{\sigma}(t)\right), v^{\sigma}(t)\right) \mathrm{d} t \\
& -\int_{[0, T)_{\mathbb{T}}}\left(B u^{\Delta}(t), v^{\sigma}(t)\right) \Delta t+\int_{[0, T)_{\mathbb{T}}}\left(B u^{\sigma}(t), v^{\Delta}(t)\right) \Delta t \\
& -\int_{[0, T)_{\mathbb{T}}}\left(B u^{\Delta}(t), v^{\sigma}(t)\right) \Delta t-\int_{[0, T)_{\mathbb{T}}}\left(B u^{\Delta}(t), v(t)\right) \Delta t,
\end{aligned}
$$

for all $H_{\Delta, T}^{1}$. 
On the other hand, by the continuity of $I_{i j}, i \in \Lambda_{1}, j \in \Lambda_{2}$, one has $\phi \in C^{1}\left(H_{T}^{1}, \mathbb{R}\right)$ and

$$
\left\langle\phi^{\prime}(u), v\right\rangle=\sum_{j=1}^{p} \sum_{i=1}^{N} I_{i j}\left(u^{i}\left(t_{j}\right)\right) v^{i}\left(t_{j}\right),
$$

for all $u, v \in H_{\Delta, T}^{1}$. Thus, $\varphi$ is continuously differentiable on $H_{\Delta, T}^{1}$ and (11) holds.

Lemma 3.2 $\phi^{\prime}$ is compact on $H_{\Delta, T}^{1}$.

Proof Let $\left\{u_{k}\right\} \subset H_{\Delta, T}^{1}$ be any bounded sequence. Since $H_{\Delta, T}^{1}$ is a Hilbert space, we can assume that $u_{k} \rightarrow u$. Theorem 2.4 implies that $\left\|u_{k}-u\right\|_{\infty} \rightarrow 0$. By (4), we have

$$
\begin{aligned}
\left\|\phi^{\prime}\left(u_{k}\right)-\phi^{\prime}(u)\right\| & =\sup _{v \in H_{\Delta, T}^{1},\|v\| \leq 1}\left|\left\langle\phi^{\prime}\left(u_{k}\right)-\phi^{\prime}(u), v\right\rangle\right| \\
& =\sup _{v \in H_{\Delta, T}^{1},\|v\| \leq 1}\left|\sum_{j=1}^{p} \sum_{i=1}^{N}\left[I_{i j}\left(u_{k}^{i}\left(t_{j}\right)\right)-I_{i j}\left(u^{i}\left(t_{j}\right)\right)\right] v^{i}\left(t_{j}\right)\right| \\
& \leq\|v\|_{\infty} \sup _{v \in H_{\Delta, T}^{1},\|v\| \leq 1}\left|\sum_{j=1}^{p} \sum_{i=1}^{N}\right| I_{i j}\left(u_{k}^{i}\left(t_{j}\right)\right)-I_{i j}\left(u^{i}\left(t_{j}\right)\right)|| \\
& \leq C_{1}\|v\| \sup _{v \in H_{\Delta, T}^{1},\|v\| \leq 1}\left|\sum_{j=1}^{p} \sum_{i=1}^{N}\right| I_{i j}\left(u_{k}^{i}\left(t_{j}\right)\right)-I_{i j}\left(u^{i}\left(t_{j}\right)\right)|| \\
& =C_{1} \sup _{v \in H_{\Delta, T}^{1},\|\nu\| \leq 1}\left|\sum_{j=1}^{p} \sum_{i=1}^{N}\right| I_{i j}\left(u_{k}^{i}\left(t_{j}\right)\right)-I_{i j}\left(u^{i}\left(t_{j}\right)\right) \mid .
\end{aligned}
$$

The continuity of $I_{i j}$ and this imply that $\phi^{\prime}\left(u_{k}\right) \rightarrow \phi^{\prime}(u)$ in $H_{\Delta, T}^{1}$. The proof is complete.

By Definition 3.1 and Lemma 3.1, the weak solutions of problem (1) correspond to the critical points of $\varphi$.

Moreover, we need more preliminaries. We define operators $G: H_{\Delta, T}^{1} \rightarrow\left(H_{\Delta, T}^{1}\right)^{*}$ as follows, for any $u \in H_{\Delta, T}^{1}$, which is given by

$$
G u(v)=\int_{[0, T)_{\mathbb{T}}}\left(B u^{\Delta}(t), v^{\sigma}(t)\right) \Delta t,
$$

for all $v \in H_{\Delta, T}^{1}$, where $\left(H_{\Delta, T}^{1}\right)^{*}$ denotes the dual space of $H_{\Delta, T}^{1}$. By the Riesz representation theorem, we can identify $\left(H_{\Delta, T}^{1}\right)^{*}$ with $H_{\Delta, T}^{1}$. Thus, $G u$ can also be viewed as a function belonging to $H_{\Delta, T}^{1}$ such that $\langle G u, v\rangle=G u(v)$ for any $u, v \in H_{\Delta, T}^{1}$ and $G$ is a bounded linear self-adjoint operator on $H_{\Delta, T}^{1}$. On the other hand, we can obtained the following lemma in the same way as the proof of Lemma 2.3 of [7].

Lemma 3.3 $G$ is compact on $H_{\Delta, T}^{1}$.

For any $u \in H_{\Delta, T}^{1}$, let

$$
q(u)=\frac{1}{2} \int_{[0, T)_{\mathbb{T}}}\left[|\dot{u}(t)|^{2}+\left(2 B u^{\sigma}(t), u^{\Delta}(t)\right)-\left(A^{\sigma}(t) u^{\sigma}(t), u^{\sigma}(t)\right)\right] \Delta t,
$$


we see that

$$
\begin{aligned}
q(u) & =\frac{1}{2}\|u\|^{2}-\frac{1}{2} \int_{[0, T)_{\mathbb{T}}}\left(\left(A^{\sigma}(t)+I_{N \times N}\right) u^{\sigma}(t)+2 B u^{\Delta}(t), u^{\sigma}(t)\right) \Delta t \\
& =\frac{1}{2}\langle(I-K) u, u\rangle,
\end{aligned}
$$

where $K: H_{\Delta, T}^{1} \rightarrow H_{\Delta, T}^{1}$ is the bounded self-adjoint linear operator defined, using Riesz representation theorem, by

$$
\langle K u, v\rangle=2\langle G u, v\rangle+\int_{[0, T)_{\mathbb{T}}}\left(\left(A^{\sigma}(t)+I_{N \times N}\right) u^{\sigma}(t), u^{\sigma}(t)\right) \Delta t, \quad \forall u, v \in H_{\Delta, T}^{1},
$$

$I_{N \times N}$ and $I$ denote $N \times N$ identity matrix and identity operator respectively. By (9), $\varphi(u)$ can be rewritten as

$$
\begin{aligned}
\varphi(u) & =q(u)+\phi(u)+J(u) \\
& =\frac{1}{2}\langle(I-K) u, u\rangle+\phi(u)+J(u) .
\end{aligned}
$$

The compact embedding of $H_{\Delta, T}^{1}$ into $C\left([0, T]_{\mathbb{T}}, R^{N}\right)$ and Lemma 3.3 imply that $K$ is compact. By classical spectral theory, we can decompose $H_{\Delta, T}^{1}$ into the orthogonal sum of invariant subspaces for $I-K$

$$
H_{\Delta, T}^{1}=H^{-} \oplus H^{0} \oplus H^{+}
$$

where $H^{0}=\operatorname{ker}(I-K)$ and $H^{-}, H^{+}$are such that, for some $\delta>0$,

$$
\begin{aligned}
& q(u) \leq-\delta\|u\|^{2}, \quad \text { if } u \in H^{-}, \\
& q(u) \geq \delta\|u\|^{2}, \quad \text { if } u \in H^{+} .
\end{aligned}
$$

Remark 3.1 $K$ has only finitely many eigenvalues $\lambda_{i}$ with $\lambda_{i}>1$ since $K$ is compact on $H_{\Delta, T}^{1}$. Hence $H^{-}$is finite dimensional. Notice that $I-K$ is a compact perturbation of the self-adjoint operator $I$. By a well-known theorem, we know that 0 is not in the essential spectrum of $I-K$. Hence $H^{0}$ is a finite dimensional space too.

To prove our main results, we need the following definitions and theorems.

Definition 3.2 ([1], p.81) Let $X$ be a real Banach space and $I \in C^{1}(X, \mathbb{R}) . I$ is said to be satisfying (PS) condition on $X$ if any sequence $\left\{x_{n}\right\} \subseteq X$ for which $I\left(x_{n}\right)$ is bounded and $I^{\prime}\left(x_{n}\right) \rightarrow 0$ as $n \rightarrow \infty$, possesses a convergent subsequence in $X$.

Definition 3.3 ([21]) Let $X$ be a real Banach space and $I \in C^{1}(X, \mathbb{R})$. $I$ is said to be satisfying (C) condition on $X$ if any sequence $\left\{x_{n}\right\} \subseteq X$ for which $I\left(x_{n}\right)$ is bounded and $\left(1+\left\|x_{n}\right\|\right) I^{\prime}\left(x_{n}\right) \rightarrow 0$ as $n \rightarrow \infty$, possesses a convergent subsequence in $X$.

Firstly, we state the local linking theorem. 
Let $X$ be a real Banach space with a direct decomposition $X=X^{1} \oplus X^{2}$. Consider two sequences of subspace

$$
X_{0}^{1} \subset X_{1}^{1} \subset \cdots \subset X^{1}, \quad X_{0}^{2} \subset X_{1}^{2} \subset \cdots \subset X^{2}
$$

such that

$$
\operatorname{dim} X_{n}^{1}<+\infty, \quad \operatorname{dim} X_{n}^{2}<+\infty, \quad n \in \mathbb{N},
$$

and

$$
X^{1}=\overline{\bigcup_{n \in \mathbb{N}} X_{n}^{1}}, \quad X^{2}=\overline{\bigcup_{n \in \mathbb{N}} X_{n}^{2}} .
$$

For every multi-index $\alpha=\left(\alpha_{1}, \alpha_{2}\right) \in \mathbb{N}^{2}$, we denote by $X_{\alpha}$ the space $X_{\alpha_{1}} \oplus X_{\alpha_{2}}$. We say $\alpha \leq \beta \Leftrightarrow \alpha_{1} \leq \beta_{1}, \alpha_{2} \leq \beta_{2}$. A sequence $\left(\alpha_{n}\right) \subset \mathbb{N}^{2}$ is admissible if for every $\alpha \in \mathbb{N}^{2}$ there is $m_{0} \in \mathbb{N}$ such that $n \geq m_{0} \Rightarrow \alpha_{n} \geq \alpha$.

Definition 3.4 (Definition 2.2, [22]) Let $I \in C^{1}(X, \mathbb{R})$. The functional $I$ satisfies condition (C)* if every sequence $\left(u_{\alpha_{n}}\right)$ such that $\alpha_{n}$ is admissible and

$$
u_{\alpha_{n}} \in X_{\alpha_{n}}, \quad \sup \left|I\left(u_{\alpha_{n}}\right)\right|<\infty, \quad\left(1+\left\|u_{\alpha_{n}}\right\|\right) I^{\prime}\left(u_{\alpha_{n}}\right) \rightarrow 0
$$

contains a subsequence which converges to a critical point of $I$.

Theorem 3.1 (Theorem 2.2, [22]) Suppose that $I \in C^{1}(X, \mathbb{R})$ satisfies the following assumptions:

$\left(\mathrm{I}_{1}\right) X^{1} \neq\{0\}$ and $I$ has a local linking at 0 with respect to $\left(X^{1}, X^{2}\right)$, that is, for some $r_{0}>0$,

$$
\begin{aligned}
& I(u) \geq 0, \quad u \in X^{1},\|u\| \leq r_{0}, \\
& I(u) \leq 0, \quad u \in X^{2},\|u\| \leq r_{0} .
\end{aligned}
$$

( $\left.\mathrm{I}_{2}\right)$ I satisfies condition $(\mathrm{C})^{*}$.

$\left(\mathrm{I}_{3}\right)$ I maps bounded sets into bounded sets.

$\left(\mathrm{I}_{4}\right)$ For every $n \in \mathbb{N}, I(u) \rightarrow-\infty$ as $\|u\| \rightarrow \infty, u \in X_{n}^{1} \oplus X^{2}$.

Then I has at least two critical points.

Remark 3.2 Since $I \in C^{1}(X, \mathbb{R})$, by condition $\left(\mathrm{I}_{1}\right)$ of Theorem 3.1, 0 is the critical point of $I$. Thus, under the conditions of Theorem 3.1, $I$ has at least one non-trivial critical point.

Secondly, we state another three critical point theorems.

Theorem 3.2 (Theorem 5.29, [23]) Let E be a Hilbert space with $E=E_{1} \oplus E_{2}$ and $E_{2}=E_{1}^{\perp}$. Suppose $I \in C^{1}(E, R)$, it satisfies (PS) condition, and

( $\left.\mathrm{I}_{5}\right) I(u)=\frac{1}{2}\langle L u, u\rangle+b(u)$, where $L u=L_{1} P_{1} u+L_{2} P_{2} u$ and $L_{\kappa}: E_{\kappa} \rightarrow E_{\kappa}$ is bounded and selfadjoint, $\kappa=1,2$, 
( $\left.\mathrm{I}_{6}\right) b^{\prime}$ is compact, and

$\left(\mathrm{I}_{7}\right)$ there exist a subspace $\widetilde{E} \subset E$ and sets $S \subset E, Q \subset \widetilde{E}$ and constants $\alpha>\omega$ such that

(i) $S \subset E_{1}$ and $\left.I\right|_{S} \geq \alpha$,

(ii) $Q$ is bounded and $\left.I\right|_{\partial Q} \leq \omega$,

(iii) $S$ and $\partial Q$ link.

Then I possesses a critical value $c \geq \alpha$.

Theorem 3.3 (Theorem 9.12, [23]) Let E be an infinite dimensional Banach space. Let $I \in$ $C^{1}(E, \mathbb{R})$ be an even functional which satisfies the $(P S)$ condition and $I(0)=0$. If $E=V \oplus W$, where $V$ is finite dimensional, and I satisfies

(I $\left.\mathrm{I}_{8}\right)$ there are constants $\rho, \alpha>0$ such that $\left.I\right|_{\partial B_{\rho} \cap W} \geq \alpha$, where $B_{\rho}=\{x \in E:\|x\|<\rho\}$,

$\left(\mathrm{I}_{9}\right)$ for each finite dimensional subspace $\widetilde{E} \subset E$, there is an $R=R(\widetilde{E})$ such that $I \leq 0$ on $\widetilde{E} \backslash B_{R(\widetilde{E})}$,

then I possesses an unbounded sequence of critical values.

Remark 3.3 As shown in [21], a deformation theorem can be proved with condition (C) replacing the usual condition (PS), and it turns out that Theorem 3.2 and Theorem 3.3 hold under condition $(C)$.

In order to state the other critical point theorem we need the following notions. Let $X$ and $Y$ be Banach spaces with $X$ being separable and reflexive, and set $E=X \oplus Y$. Let $\mathcal{S} \subset X^{*}$ be a dense subset. For each $s \in \mathcal{S}$ there is a semi-norm on $E$ defined by

$$
p_{s}: E \rightarrow R, \quad p_{s}(u)=|s(x)|+\|y\| \quad \text { for } u=x+y \in X \oplus Y .
$$

We denote by $\mathcal{T}_{\mathcal{S}}$ the topology on $E$ induced by semi-norm family $\left\{p_{s}\right\}$, and let $w$ and $w^{*}$ denote the weak-topology and weak ${ }^{*}$-topology, respectively.

For a functional $\Phi \in C^{1}(E, R)$ we write $\Phi_{a}=\{u \in E: \Phi(u) \geq a\}$. Recall that $\Phi^{\prime}$ is said to be weak sequentially continuous if for any $u_{k} \rightarrow u$ in $E$ one has $\lim _{k \rightarrow \infty} \Phi^{\prime}\left(u_{k}\right) v \rightarrow$ $\Phi^{\prime}(u) v$ for each $v \in E$, i.e. $\Phi^{\prime}:(E, w) \rightarrow\left(E^{*}, w^{*}\right)$ is sequentially continuous. For $c \in R$ we say that $\Phi$ satisfies condition $(C)_{c}$ if any sequence $\left\{u_{k}\right\} \subset E$ such that $\Phi\left(u_{k}\right) \rightarrow c$ and $(1+$ $\left.\left\|u_{k}\right\|\right) \Phi^{\prime}\left(u_{k}\right) \rightarrow 0$ as $k \rightarrow \infty$ contains a convergent subsequence.

Suppose that

$\left(\Phi_{0}\right)$ for any $c \in R, \Phi_{c}$ is $\mathcal{T}_{\mathcal{S}}$-closed, and $\Phi^{\prime}:\left(\Phi_{c}, \mathcal{T}_{\mathcal{S}}\right) \rightarrow\left(E^{*}, w^{*}\right)$ is continuous;

$\left(\Phi_{1}\right)$ there exists $\rho>0$ such that $\kappa:=\inf \Phi\left(\partial B_{\rho} \cap Y\right)>0$, where

$$
B_{\rho}=\{u \in E:\|u\|<\rho\}
$$

$\left(\Phi_{2}\right)$ there exist a finite dimensional subspace $Y_{0} \subset Y$ and $R>\rho$ such that $\bar{c}:=\sup \Phi\left(E_{0}\right)<$ $\infty$ and $\sup \Phi\left(E_{0} \backslash S_{0}\right)<\inf \Phi\left(B_{\rho} \cap Y\right)$, where

$$
E_{0}:=X \oplus Y_{0}, \quad \text { and } \quad S_{0}=\left\{u \in E_{0}:\|u\| \leq R\right\}
$$

Theorem 3.4 ([24]) Assume that $\Phi$ is even and $\left(\Phi_{0}\right)-\left(\Phi_{2}\right)$ are satisfied. Then $\Phi$ has at least $m=\operatorname{dim} Y_{0}$ pairs of critical points with critical values less than or equal to $\bar{c}$ provided $\Phi$ satisfies condition $(\mathrm{C})_{c}$, for all $c \in[\kappa, \bar{c}]$. 
Remark 3.4 In our applications we take $\mathcal{S}=X^{*}$, so that $\mathcal{T}_{\mathcal{S}}$ is the product topology on $E=X \oplus Y$ given by the weak topology on $X$ and the strong topology on $Y$.

\section{Main results}

First of all, we give two existence results.

Theorem 4.1 Suppose that (A) and the following conditions are satisfied.

(F $\mathrm{F}_{1} \lim _{|x| \rightarrow \infty} \frac{F(t, x)}{|x|^{2}}=+\infty$ uniformly for $\Delta$-a.e. $t \in[0, T]_{\mathbb{T}}$,

$\left(\mathrm{F}_{2}\right) \lim _{|x| \rightarrow 0} \frac{F(t, x)}{|x|^{2}}=0$ uniformly for $\Delta$-a.e. $t \in[0, T]_{\mathbb{T}}$,

$\left(\mathrm{F}_{3}\right)$ there exist $\lambda>2$ and $\eta>\lambda-2$ such that

$$
\limsup _{|x| \rightarrow \infty} \frac{F(t, x)}{|x|^{\lambda}}<\infty \quad \text { uniformly for } \Delta \text {-a.e. } t \in[0, T]_{\mathbb{T}}
$$

and

$$
\liminf _{|x| \rightarrow \infty} \frac{(\nabla F(t, x), x)-2 F(t, x)}{|x|^{\eta}}>0 \quad \text { uniformly for } \Delta \text {-a.e. } t \in[0, T]_{\mathbb{T}},
$$

$\left(\mathrm{F}_{4}\right)$ there exists $r>0$ such that

$$
F(t, x) \geq 0, \quad \forall|x| \leq r \text {, and } \Delta \text {-a.e. } t \in[0, T]_{\mathbb{T}},
$$

$\left(\mathrm{F}_{5}\right)$ there exist $\beta_{i j}, \gamma_{i j}>0$, and $\xi_{i j} \in[0,1)$ such that

$$
\left|I_{i j}(t)\right| \leq \beta_{i j}+\gamma_{i j}|t|^{\xi_{i j}} \quad \text { for every } t \in \mathbb{R}, i \in \Lambda_{1}, j \in \Lambda_{2}
$$

( $\left.\mathrm{F}_{6}\right) \int_{0}^{t} I_{i j}(s) \mathrm{d} s \leq 0$ for every $t \in \mathbb{R}, i \in \Lambda_{1}, j \in \Lambda_{2}$,

$\left(\mathrm{F}_{7}\right)$ there exists $\zeta_{i j}>0$ such that

$$
2 \int_{0}^{t} I_{i j}(s) \mathrm{d} s-I_{i j}(t) t \geq 0, \quad \text { for all } i \in \Lambda_{1}, j \in \Lambda_{2} \text { and }|t| \geq \zeta_{i j}
$$

and

$$
\lim _{t \rightarrow 0} \frac{I_{i j}(t)}{t}=0, \quad \text { for all } i \in \Lambda_{1}, j \in \Lambda_{2} .
$$

Then problem (1) has at least two weak solutions. The one is a nontrivial weak solution, the other is trivial weak solution.

In order to prove Theorem 4.1, we prove the following lemma.

Lemma 4.1 Assume that $(\mathrm{A}),\left(\mathrm{F}_{3}\right),\left(\mathrm{F}_{5}\right)$, and $\left(\mathrm{F}_{7}\right)$ are satisfied, then $\varphi$ satisfies condi$\operatorname{tion}(\mathrm{C})^{*}$.

Proof Let $\left\{u_{\alpha_{n}}\right\}$ be a sequence in $H_{\Delta, T}^{1}$ such that $\alpha_{n}$ is admissible and

$$
u_{\alpha_{n}} \in X_{\alpha_{n}}, \quad \sup \left|\varphi\left(u_{\alpha_{n}}\right)\right|<+\infty, \quad\left(1+\left\|u_{\alpha_{n}}\right\|\right) \varphi^{\prime}\left(u_{\alpha_{n}}\right) \rightarrow 0,
$$


then there exists a constant $C_{2}>0$ such that

$$
\left|\varphi\left(u_{\alpha_{n}}\right)\right| \leq C_{2}, \quad\left(1+\left\|u_{\alpha_{n}}\right\|\right)\left\|\varphi^{\prime}\left(u_{\alpha_{n}}\right)\right\| \leq C_{2}
$$

for all large $n$. On the other hand, by $\left(\mathrm{F}_{3}\right)$, there are constants $C_{3}>0$ and $\rho_{1}>0$ such that

$$
F(t, x) \leq C_{3}|x|^{\lambda}
$$

for all $|x| \geq \rho_{1}$ and $\Delta$-a.e. $t \in[0, T]_{\mathbb{T}}$. By (A) one has

$$
|F(t, x)| \leq \max _{s \in\left[0, \rho_{1}\right]} a(s) b(t)
$$

for all $|x| \leq \rho_{1}$ and a.e. $t \in[0, T]$. It follows from (16) and (17) that

$$
|F(t, x)| \leq \max _{s \in\left[0, \rho_{1}\right]} a(s) b(t)+C_{3}|x|^{\lambda},
$$

for all $x \in \mathbb{R}^{N}$ and $\Delta$-a.e. $t \in[0, T]_{\mathbb{T}}$. Since $\bar{a}_{l m} \in L^{\infty}\left([0, T)_{\mathbb{T}}, \mathbb{R}\right)$ for all $l, m=1,2, \ldots, N$, there exists a constant $C_{4} \geq 1$ such that

$$
\left|\int_{[0, T)_{\mathbb{T}}}\left(A^{\sigma}(t) u^{\sigma}(t), u^{\sigma}(t)\right) \Delta t\right| \leq C_{4} \int_{[0, T)_{\mathbb{T}}}\left|u^{\sigma}(t)\right|^{2} \Delta t, \quad \forall u \in H_{\Delta, T}^{1} .
$$

Let $\bar{b}=\max _{l, m=1,2, \ldots, N}\left\{\bar{b}_{l m}\right\}$. For $\forall u \in H_{\Delta, T}^{1}$, we have

$$
\begin{aligned}
\left|\int_{[0, T)_{\mathbb{T}}}\left(B u^{\sigma}(t), u^{\Delta}(t)\right) \Delta t\right| & \leq \frac{1}{2} \int_{[0, T)_{\mathbb{T}}}\left|2 B u^{\sigma}(t)\right|\left|u^{\Delta}(t)\right| \Delta t \\
& \leq \frac{1}{4} \int_{[0, T)_{\mathbb{T}}}\left[\left|2 B u^{\sigma}(t)\right|^{2}+\left|u^{\Delta}(t)\right|^{2}\right] \Delta t \\
& \leq \frac{1}{2} \bar{b} N \int_{[0, T)_{\mathbb{T}}}\left|u^{\sigma}(t)\right|^{2} \Delta t+\frac{1}{4} \int_{[0, T)_{\mathbb{T}}}\left|u^{\Delta}(t)\right|^{2} \Delta t .
\end{aligned}
$$

From $\left(\mathrm{F}_{5}\right)$ and $(10)$, we have

$$
\begin{aligned}
|\phi(u)| & \leq \sum_{j=1}^{p} \sum_{i=1}^{N} \int_{0}^{\left|u^{i}\left(t_{j}\right)\right|}\left(\beta_{i j}+\gamma_{i j}|t|^{\xi_{i j}}\right) \mathrm{d} t \\
& \leq \bar{\beta} p N\|u\|_{\infty}+\bar{\gamma} \sum_{j=1}^{p} \sum_{i=1}^{N}\|u\|_{\infty}^{\xi_{i j}+1} \\
& \leq \bar{\beta} p N C_{1}\|u\|+\bar{\gamma} C_{1}^{\xi_{i j}+1} \sum_{j=1}^{p} \sum_{i=1}^{N}\|u\|^{\xi_{i j}+1}
\end{aligned}
$$

for all $u \in H_{\Delta, T}^{1}$, where $\bar{\beta}=\max _{i \in \Lambda_{1}, j \in \Lambda_{2}}\left\{\beta_{i j}\right\}, \bar{\gamma}=\max _{i \in \Lambda_{1}, j \in \Lambda_{2}}\left\{\gamma_{i j}\right\}$. Combining (18), (19), (20), (21), and Hölder's inequality, we have

$$
\begin{aligned}
\frac{1}{2}\left\|u_{\alpha_{n}}\right\|^{2}= & \varphi\left(u_{\alpha_{n}}\right)-\phi\left(u_{\alpha_{n}}\right)+\frac{1}{2} \int_{[0, T)_{\mathbb{T}}}\left|u_{\alpha_{n}}(t)\right|^{2} \Delta t+\frac{1}{2} \int_{[0, T)_{\mathbb{T}}}\left(A^{\sigma}(t) u_{\alpha_{n}}(t), u_{\alpha_{n}}^{\sigma}(t)\right) \Delta t \\
& -\int_{[0, T)_{\mathbb{T}}}\left(B u_{\alpha_{n}}^{\sigma}(t), u_{\alpha_{n}}^{\Delta}(t)\right) \mathrm{d} t-J(u)
\end{aligned}
$$




$$
\begin{aligned}
\leq & C_{2}+\bar{\beta} p N C_{1}\left\|u_{\alpha_{n}}\right\|+\bar{\gamma} C_{1}^{\xi_{i j}+1} \sum_{j=1}^{p} \sum_{i=1}^{N}\left\|u_{\alpha_{n}}\right\| \|^{\xi_{i j}+1}+C_{4} \int_{[0, T)_{\mathbb{T}}}\left|u_{\alpha_{n}}^{\sigma}(t)\right|^{2} \Delta t \\
& +\frac{1}{2} \bar{b} N \int_{[0, T)_{\mathbb{T}}}\left|u_{\alpha_{n}}^{\sigma}(t)\right|^{2} \Delta t+\frac{1}{4} \int_{[0, T)_{\mathbb{T}}}\left|u_{\alpha_{n}}^{\Delta}(t)\right|^{2} \Delta t \\
& +C_{3} \int_{[0, T)_{\mathbb{T}}}\left|u_{\alpha_{n}}^{\sigma}(t)\right|^{\lambda} \Delta t+\max _{s \in\left[0, \rho_{1}\right]} a(s) \int_{[0, T)_{\mathbb{T}}} b^{\sigma}(t) \Delta t \\
\leq & C_{2}+\bar{\beta} p N C_{1}\left\|u_{\alpha_{n}}\right\|+\bar{\gamma} C_{1}^{\xi_{i j}} \sum_{j=1}^{p} \sum_{i=1}^{N}\left\|u_{\alpha_{n}}\right\|\left\|^{\xi_{i j}+1}+\frac{1}{4}\right\| u_{\alpha_{n}} \|^{2} \\
& +\left(C_{4}+\frac{1}{2} \bar{b} N\right) T^{\frac{\lambda-2}{\lambda}}\left(\int_{[0, T)_{\mathbb{T}}}\left|u_{\alpha_{n}}^{\sigma}(t)\right|^{\lambda} \Delta t\right)^{\frac{2}{\lambda}} \\
& +C_{3} \int_{[0, T)_{\mathbb{T}}}\left|u_{\alpha_{n}}^{\sigma}(t)\right|^{\lambda} \Delta t+C_{5},
\end{aligned}
$$

for all large $n$, where $C_{5}=\max _{s \in\left[0, \rho_{1}\right]} a(s) \int_{[0, T)_{\mathbb{T}}} b^{\sigma}(t) \Delta t$. On the other hand, by $\left(\mathrm{F}_{3}\right)$, there exist $C_{6}>0$ and $\rho_{2}>0$ such that

$$
(\nabla F(t, x), x)-2 F(t, x) \geq C_{6}|x|^{\eta}
$$

for all $|x| \geq \rho_{2}$ and $\Delta$-a.e. $t \in[0, T]_{\mathbb{T}}$. By (A), we have

$$
|(\nabla F(t, x), x)-2 F(t, x)| \leq C_{7} b(t)
$$

for all $|x| \leq \rho_{2}$ and $\Delta$-a.e. $t \in[0, T]_{\mathbb{T}}$, where $C_{7}=\left(2+\rho_{2}\right) \max _{s \in\left[0, \rho_{2}\right]} a(s)$. Combining (23) and (24), one has

$$
(\nabla F(t, x), x)-2 F(t, x) \geq C_{6}|x|^{\eta}-C_{6} \rho_{2}^{\eta}-C_{7} b(t)
$$

for all $x \in \mathbb{R}^{N}$ and $\Delta$-a.e. $t \in[0, T]_{\mathbb{T}}$. According to $\left(\mathrm{F}_{7}\right)$, there exists $C_{8}>0$ such that

$$
2 \int_{0}^{t} I_{i j}(s) \mathrm{d} s-I_{i j}(t) t \geq-C_{8}, \quad \text { for all } i \in \Lambda_{1}, j \in \Lambda_{2} \text { and } t \in \mathbb{R} .
$$

Thus by (15), (25), and (26), we obtain

$$
\begin{aligned}
3 C_{2} \geq & 2 \varphi\left(u_{\alpha_{n}}\right)-\left\langle\varphi^{\prime}\left(u_{\alpha_{n}}\right), u_{\alpha_{n}}\right\rangle \\
= & 2 \phi\left(u_{\alpha_{n}}\right)-\left\langle\phi^{\prime}\left(u_{\alpha_{n}}\right), u_{\alpha_{n}}\right\rangle \\
& +\int_{[0, T)_{\mathbb{T}}}\left[\left(\nabla F\left(\sigma(t), u_{\alpha_{n}}^{\sigma}(t)\right), u_{\alpha_{n}}^{\sigma}(t)\right)-2 F\left(\sigma(t), u_{\alpha_{n}}^{\sigma}(t)\right)\right] \Delta t \\
& +\int_{[0, T)_{\mathbb{T}}}\left(B u_{\alpha_{n}}^{\Delta}(t), u_{\alpha_{n}}(t)\right) \Delta t-\int_{[0, T)_{\mathbb{T}}}\left(B u_{\alpha_{n}}^{\Delta}(t), u_{\alpha_{n}}^{\sigma}(t)\right) \Delta t \\
= & \sum_{j=1}^{p} \sum_{i=1}^{N}\left(2 \int_{0}^{u_{\alpha_{n}}^{i}\left(t_{j}\right)} I_{i j}(t) \mathrm{d} t-I_{i j}\left(u_{\alpha_{n}}^{i}\left(t_{j}\right)\right) u_{\alpha_{n}}^{i}\left(t_{j}\right)\right) \\
& +\int_{[0, T)_{\mathbb{T}}}\left[\left(\nabla F\left(\sigma(t), u_{\alpha_{n}}^{\sigma}(t)\right), u_{\alpha_{n}}^{\sigma}(t)\right)-2 F\left(\sigma(t), u_{\alpha_{n}}^{\sigma}(t)\right)\right] \Delta t
\end{aligned}
$$




$$
\begin{aligned}
& +\int_{[0, T)_{\mathbb{T}}}\left(B u_{\alpha_{n}}^{\Delta}(t), u_{\alpha_{n}}(t)\right) \Delta t-\int_{[0, T)_{\mathbb{T}}}\left(B u_{\alpha_{n}}^{\Delta}(t), u_{\alpha_{n}}(t)+\mu(t) u_{\alpha_{n}}^{\Delta}(t)\right) \Delta t \\
= & \sum_{j=1}^{p} \sum_{i=1}^{N}\left(2 \int_{0}^{u_{\alpha_{n}}^{i}\left(t_{j}\right)} I_{i j}(t) \mathrm{d} t-I_{i j}\left(u_{\alpha_{n}}^{i}\left(t_{j}\right)\right) u_{\alpha_{n}}^{i}\left(t_{j}\right)\right) \\
& +\int_{[0, T)_{\mathbb{T}}}\left[\left(\nabla F\left(\sigma(t), u_{\alpha_{n}}^{\sigma}(t)\right), u_{\alpha_{n}}^{\sigma}(t)\right)-2 F\left(\sigma(t), u_{\alpha_{n}}^{\sigma}(t)\right)\right] \Delta t \\
& -\int_{[0, T)_{\mathbb{T}}} \mu(t)\left(B u_{\alpha_{n}}^{\Delta}(t), u_{\alpha_{n}}^{\Delta}(t)\right) \Delta t \\
= & \sum_{j=1}^{p} \sum_{i=1}^{N}\left(2 \int_{0}^{u_{\alpha_{n}}^{i}\left(t_{j}\right)} I_{i j}(t) \mathrm{d} t-I_{i j}\left(u_{\alpha_{n}}^{i}\left(t_{j}\right)\right) u_{\alpha_{n}}^{i}\left(t_{j}\right)\right) \\
& +\int_{[0, T)_{\mathbb{T}}}\left[\left(\nabla F\left(\sigma(t), u_{\alpha_{n}}^{\sigma}(t)\right), u_{\alpha_{n}}^{\sigma}(t)\right)-2 F\left(\sigma(t), u_{\alpha_{n}}^{\sigma}(t)\right)\right] \Delta t \\
\geq & -p N C_{8}+C_{6} \int_{[0, T)_{\mathbb{T}}}\left|u_{\alpha_{n}}^{\sigma}\right|^{\eta} \Delta t-C_{6} \rho_{2}^{\eta} T-C_{7} \int_{[0, T)_{\mathbb{T}}} b^{\sigma}(t) \Delta t,
\end{aligned}
$$

for all large $n$. From (27), $\int_{[0, T)_{\mathbb{T}}}\left|u_{\alpha_{n}}^{\sigma}\right|^{\eta} \Delta t$ is bounded. If $\eta>\lambda$, by Hölder's inequality, we have

$$
\int_{[0, T)_{\mathbb{T}}}\left|u_{\alpha_{n}}^{\sigma}\right|^{\lambda} \Delta t \leq T^{\frac{\eta-\lambda}{\eta}}\left(\int_{[0, T)_{\mathbb{T}}}\left|u_{\alpha_{n}}^{\sigma}\right|^{\eta} \Delta t\right)^{\frac{\lambda}{\eta}} .
$$

Since $\xi_{i j} \in[0,1)$, for all $i \in \Lambda_{1}, j \in \Lambda_{2}$, by (22) and (28), $\left\{u_{\alpha_{n}}\right\}$ is bounded in $H_{\Delta, T}^{1}$. If $\eta \leq \lambda$, by (7), we obtain

$$
\begin{aligned}
\int_{[0, T)_{\mathbb{T}}}\left|u_{\alpha_{n}}^{\sigma}(t)\right|^{\lambda} \Delta t & =\int_{[0, T)_{\mathbb{T}}}\left|u_{\alpha_{n}}^{\sigma}(t)\right|^{\eta}\left|u_{\alpha_{n}}^{\sigma}(t)\right|^{\lambda-\eta} \Delta t \\
& \leq\left\|u_{\alpha_{n}}\right\|_{\infty}^{\lambda-\eta} \int_{[0, T)_{\mathbb{T}}}\left|u_{\alpha_{n}}^{\sigma}(t)\right|^{\eta} \Delta t \\
& \leq C_{1}^{\lambda-\eta}\left\|u_{\alpha_{n}}\right\|^{\lambda-\eta} \int_{[0, T)_{\mathbb{T}}}\left|u_{\alpha_{n}}^{\sigma}(t)\right|^{\eta} \Delta t .
\end{aligned}
$$

Since $\xi_{i j} \in[0,1), \lambda-\eta<2$, by (22) and (29), $\left\{u_{\alpha_{n}}\right\}$ is also bounded in $H_{\Delta, T}^{1}$. Hence, $\left\{u_{\alpha_{n}}\right\}$ is also bounded in $H_{\Delta, T}^{1}$. Going if necessary to a subsequence, we can assume that $u_{\alpha_{n}} \rightarrow u$ in $H_{\Delta, T}^{1}$. From Theorem 2.4, we have $\left\|u_{\alpha_{n}}-u\right\|_{\infty} \rightarrow 0$ and $\int_{[0, T)_{T}}\left|u_{\alpha_{n}}^{\sigma}-u^{\sigma}\right|^{2} \Delta t \rightarrow 0$. Thus, by (19) and (20), we have

$$
\begin{aligned}
& \int_{[0, T)_{\mathbb{T}}}\left|u_{\alpha_{n}}^{\Delta}-u^{\Delta}\right|^{2} \Delta t \\
& =\left\langle\varphi^{\prime}\left(u_{\alpha_{n}}\right)-\varphi^{\prime}(u), u_{\alpha_{n}}-u\right\rangle-\sum_{j=1}^{p} \sum_{i=1}^{N}\left(I_{i j}\left(u_{\alpha_{n}}^{i}\left(t_{j}\right)\right)-I_{i j}\left(u^{i}\left(t_{j}\right)\right)\right)\left(u_{\alpha_{n}}^{i}\left(t_{j}\right)-u^{i}\left(t_{j}\right)\right) \\
& \quad+\int_{[0, T)_{\mathbb{T}}}\left(A^{\sigma}(t)\left(u_{\alpha_{n}}^{\sigma}-u\right), u_{\alpha_{n}}^{\sigma}-u^{\sigma}\right) \Delta t+2 \int_{[0, T)_{\mathbb{T}}}\left(B\left(u_{\alpha_{n}}^{\Delta}-u^{\Delta}\right), u_{\alpha_{n}}-u\right) \Delta t \\
& \quad+\int_{[0, T)_{\mathbb{T}}}\left(\nabla F\left(\sigma(t), u_{\alpha_{n}}^{\sigma}\right)-\nabla F\left(\sigma(t), u^{\sigma}\right), u_{\alpha_{n}}^{\sigma}-u^{\sigma}\right) \Delta t
\end{aligned}
$$




$$
\begin{aligned}
\leq & \left\|\varphi^{\prime}\left(u_{\alpha_{n}}\right)\right\|\left\|u_{\alpha_{n}}-u\right\|-\left\langle\varphi^{\prime}(u), u_{\alpha_{n}}-u\right\rangle \\
& -\sum_{j=1}^{p} \sum_{i=1}^{N}\left(I_{i j}\left(u_{\alpha_{n}}^{i}\left(t_{j}\right)\right)-I_{i j}\left(u^{i}\left(t_{j}\right)\right)\right)\left(u_{\alpha_{n}}^{i}\left(t_{j}\right)-u^{i}\left(t_{j}\right)\right) \\
& +C_{4} \int_{[0, T)_{\mathbb{T}}}\left|u_{\alpha_{n}}^{\sigma}-u^{\sigma}\right|^{2} \Delta t+\frac{1}{2} \bar{b} N \int_{[0, T)_{\mathbb{T}}}\left|u_{\alpha_{n}}^{\sigma}-u^{\sigma}\right|^{2} \Delta t+\frac{1}{4} \int_{[0, T)_{\mathbb{T}}}\left|u_{\alpha_{n}}^{\Delta}-u^{\Delta}\right|^{2} \Delta t \\
& +\left\|u_{\alpha_{n}}-u\right\|_{\infty} \int_{[0, T)_{\mathbb{T}}}\left|\nabla F\left(\sigma(t), u_{\alpha_{n}}^{\sigma}\right)-\nabla F\left(\sigma(t), u^{\sigma}\right)\right| \Delta t .
\end{aligned}
$$

This implies $\int_{[0, T)_{T}}\left|\dot{u}_{\alpha_{n}}-\dot{u}\right|^{2} \Delta t \rightarrow 0$, and hence $\left\|u_{\alpha_{n}}-u\right\| \rightarrow 0$. Therefore, $u_{\alpha_{n}} \rightarrow u$ in $H_{\Delta, T}^{1}$. Hence $\varphi$ satisfies condition $(\mathrm{C})^{*}$.

Now, we prove Theorem 3.1.

Proof By Lemma 3.1, $\varphi \in C^{1}(X, \mathbb{R})$. Set $X=H_{\Delta, T}^{1}, X^{1}=H^{+}$with $\left(e_{n}\right)_{n \geq 1}$ being its Hilbert basis, $X^{2}=H^{-} \oplus H^{0}$, and define

$$
\begin{aligned}
& X_{n}^{1}=\operatorname{span}\left\{e_{1}, e_{2}, \ldots, e_{n}\right\}, \quad n \in \mathbb{N}, \\
& X_{n}^{2}=X^{2}, \quad n \in \mathbb{N} .
\end{aligned}
$$

Then we have

$$
X_{0}^{1} \subset X_{1}^{1} \subset \cdots \subset X^{1}, \quad X_{0}^{2} \subset X_{1}^{2} \subset \cdots \subset X^{2}, \quad X^{1}=\overline{\bigcup_{n \in \mathbb{N}} X_{n}^{1}}, \quad X^{2}=\overline{\bigcup_{n \in \mathbb{N}} X_{n}^{2}},
$$

and

$$
\operatorname{dim} X_{n}^{1}<+\infty, \quad \operatorname{dim} X_{n}^{2}<+\infty, \quad n \in \mathbb{N} .
$$

We divide our proof into four parts in order to show Theorem 3.1.

Firstly, by Lemma 4.1, $\varphi$ satisfies condition $(C)^{*}$.

Secondly, we show that $\varphi$ maps bounded sets into bounded sets.

It follows from (9), (18), (19), (20), and (21) that

$$
\begin{aligned}
|\varphi(u)|= & \left.\left|\frac{1}{2} \int_{[0, T)_{\mathbb{T}}}\right| u^{\Delta}(t)\right|^{2} \Delta d t+\sum_{j=1}^{p} \sum_{i=1}^{N} \int_{0}^{u^{i}\left(t_{j}\right)} I_{i j}(t) \mathrm{d} t+\int_{[0, T)_{\mathbb{T}}}\left(B u^{\sigma}(t), u^{\Delta}(t)\right) \Delta t \mid \\
& -\frac{1}{2} \int_{[0, T)_{\mathbb{T}}}\left(A^{\sigma}(t) u^{\sigma}(t), u^{\sigma}(t)\right) \Delta t+J(u) \\
\leq & \frac{1}{2} \int_{[0, T)_{\mathbb{T}}}\left|u^{\Delta}(t)\right|^{2} \Delta t+\frac{C_{4}}{2} \int_{[0, T)_{\mathbb{T}}}\left|u^{\sigma}(t)\right|^{2} t+\bar{\beta} p N C_{1}\|u\| \\
& +\bar{\gamma} C_{1}^{\xi_{i j}+1} \sum_{j=1}^{p} \sum_{i=1}^{N}\|u\|^{\xi_{i j}+1} \\
& +\frac{1}{2} \bar{b} N \int_{[0, T)_{\mathbb{T}}}\left|u^{\sigma}(t)\right|^{2} \Delta t+\frac{1}{4} \int_{[0, T)_{\mathbb{T}}}\left|u^{\Delta}(t)\right|^{2} \Delta t+C_{3} \int_{[0, T)_{\mathbb{T}}}\left|u^{\sigma}(t)\right|^{\lambda} \Delta t \\
& +\max _{s \in\left[0, \rho_{1}\right]} a(s) \int_{[0, T)_{\mathbb{T}}} b^{\sigma}(t) \Delta t
\end{aligned}
$$




$$
\begin{aligned}
\leq & \frac{1}{2}\left(C_{4} C_{1}^{2}+\bar{b} N C_{1}^{2}+2\right)\|u\|^{2}+\bar{\beta} p N C_{1}\|u\| \\
& +\bar{\gamma} C_{1}^{\xi_{i j}+1} \sum_{j=1}^{p} \sum_{i=1}^{N}\|u\|^{\xi_{i j}+1}+C_{3} T\|u\|_{\infty}^{\lambda}+C_{5} \\
\leq & \frac{1}{2}\left(C_{4} C_{1}^{2}+\bar{b} N C_{1}^{2}+2\right)\|u\|^{2}+\bar{\beta} p N C_{1}\|u\| \\
& +\bar{\gamma} C_{1}^{\xi_{i j}+1} \sum_{j=1}^{p} \sum_{i=1}^{N}\|u\|^{\xi_{i j}+1}+C_{3} T C_{1}^{\lambda}\|u\|^{\lambda}+C_{5},
\end{aligned}
$$

for all $u \in H_{\Delta, T}^{1}$. Thus, $\varphi$ maps bounded sets into bounded sets.

Thirdly, we claim that $\varphi$ has a local linking at 0 with respect to $\left(X^{1}, X^{2}\right)$.

Applying $\left(\mathrm{F}_{2}\right)$, for $\epsilon_{1}=\frac{\delta}{4 C_{1}^{2}}$, there exists $\rho_{3}>0$ such that

$$
|F(t, x)| \leq \epsilon_{1}|x|^{2}
$$

for all $|x| \leq \rho_{3}$ and $\Delta$-a.e. $t \in[0, T]_{\mathbb{T}}$. By $\left(\mathrm{F}_{7}\right)$, for $\epsilon_{2}=\frac{\delta}{4 p N C_{1}^{2}}$, there exists $\rho_{4}>0$ such that

$$
\left|I_{i j}(t)\right| \leq \epsilon_{2}|t|, \quad|t| \leq \rho_{4}, i \in \Lambda_{1}, j \in \Lambda_{2} .
$$

Let $\rho_{5}=\min \left\{\rho_{3}, \rho_{4}\right\}$. For $u \in X^{1}$ with $\|u\| \leq r_{1} \triangleq \frac{\rho_{5}}{C_{1}}$, by (7), (9), (14), (30), and (31), we have

$$
\begin{aligned}
\varphi(u) & =q(u)+\sum_{j=1}^{p} \sum_{i=1}^{N} \int_{0}^{u^{i}\left(t_{j}\right)} I_{i j}(t) \mathrm{d} t-\int_{[0, T)_{\mathbb{T}}} F\left(\sigma(t), u^{\sigma}(t)\right) \Delta t \\
& \geq \delta\|u\|^{2}-\sum_{j=1}^{p} \sum_{i=1}^{N} \int_{0}^{\left|u^{i}\left(t_{j}\right)\right|}\left|I_{i j}(t)\right| \mathrm{d} t-\epsilon_{1} \int_{[0, T)_{\mathbb{T}}}\left|u^{\sigma}(t)\right|^{2} \Delta t \\
& \geq \delta\|u\|^{2}-\sum_{j=1}^{p} \sum_{i=1}^{N} \int_{0}^{\left|u^{i}\left(t_{j}\right)\right|} \epsilon_{2}|t| \mathrm{d} t-\epsilon_{1} \int_{[0, T)_{\mathbb{T}}}\left|u^{\sigma}(t)\right|^{2} \Delta t \\
& \geq \delta\|u\|^{2}-\epsilon_{2} \sum_{j=1}^{p} \sum_{i=1}^{N}\|u\|_{\infty}^{2}-\epsilon_{1} \int_{[0, T)_{\mathbb{T}}}\left|u^{\sigma}(t)\right|^{2} \Delta t \\
& \geq \delta\|u\|^{2}-\epsilon_{2} p N C_{1}^{2}\|u\|^{2}-\epsilon_{1} C_{1}^{2}\|u\|^{2} \\
& \geq \delta\|u\|^{2}-\frac{\delta}{4}\|u\|^{2}-\frac{\delta}{4}\|u\|^{2} \\
& =\frac{\delta}{2}\|u\|^{2} .
\end{aligned}
$$

This implies that

$$
\varphi(u) \geq 0, \quad \forall u \in X^{1} \text { with }\|u\| \leq r_{1} .
$$

On the other hand, it follows from $\left(\mathrm{F}_{6}\right)$ that

$$
\phi(u) \leq 0,
$$


for all $u \in H_{T}^{1}$. Let $u=u^{-}+u^{0} \in X^{2}$ satisfies $\|u\| \leq r_{2} \triangleq \frac{r}{C_{1}}$, using (F $\left.\mathrm{F}_{4}\right)$, (7), (9), (13), and (32), we obtain

$$
\begin{aligned}
\varphi(u) & =q(u)+\phi(u)-\int_{[0, T)_{\mathbb{T}}} F\left(\sigma(t), u^{\sigma}(t)\right) \Delta t \\
& \leq-\delta\left\|u^{-}\right\|^{2}-\int_{[0, T)_{\mathbb{T}}} F\left(\sigma(t), u^{\sigma}(t)\right) \Delta t \\
& \leq-\delta\left\|u^{-}\right\|^{2} .
\end{aligned}
$$

This implies that

$$
\varphi(u) \leq 0, \quad \forall u \in X^{2} \text { with }\|u\| \leq r_{2} .
$$

Let $r_{0}=\min \left\{r_{1}, r_{2}\right\}$. Then $\varphi$ satisfies the condition $\left(\mathrm{I}_{1}\right)$ of Theorem 3.1.

Finally, we claim that, for every $n \in \mathbb{N}$,

$$
\varphi(u) \rightarrow-\infty \quad \text { as }\|u\| \rightarrow \infty, u \in X_{n}^{1} \oplus X^{2} .
$$

For given $n \in \mathbb{N}$, since $X_{n}^{1} \oplus X^{2}$ is a finite-dimensional space, there exists $C_{9}>0$ such that

$$
\|u\| \leq C_{9}\|u\|_{L_{\Delta}^{2}}, \quad \forall u \in X_{n}^{1} \oplus X^{2} .
$$

By $\left(\mathrm{F}_{1}\right)$, there exists $\rho_{6}>0$ such that

$$
F(t, x) \geq C_{9}^{2}\left(C_{4} C_{1}^{2}+\bar{b} N C_{1}^{2}+2+\delta\right)|x|^{2},
$$

for all $|x| \geq \rho_{6}$ and $\Delta$-a.e. $t \in[0, T]_{\mathbb{T}}$. From (A), we get

$$
|F(t, x)| \leq \max _{s \in\left[0, \rho_{6}\right]} a(s) b(t)
$$

for all $|x| \leq \rho_{6}$ and $\Delta$-a.e. $t \in[0, T]_{\mathbb{T}}$. Equations (34) and (35) imply that

$$
F(t, x) \geq C_{9}^{2}\left(C_{4} C_{1}^{2}+\bar{b} N C_{1}^{2}+2+\delta\right)|x|^{2}-C_{10}-\max _{s \in\left[0, \rho_{6}\right]} a(s) b(t),
$$

for all $x \in \mathbb{R}^{N}$ and $\Delta$-a.e. $t \in[0, T]_{\mathbb{T}}$, where $C_{10}=C_{9}^{2}\left(C_{4}+\frac{\bar{b} N}{2}+\frac{1}{2}+\delta\right) \rho_{6}^{2}$. Using (9), (13), (19), (21), (32), and (36), we have, for $u=u^{+}+u^{0}+u^{-} \in X_{n}^{1} \oplus X^{2}=X_{n}^{1} \oplus H^{0} \oplus H^{-}$,

$$
\begin{aligned}
\varphi(u)= & \frac{1}{2} \int_{[0, T)_{\mathbb{T}}}\left|u^{\Delta}(t)\right|^{2} \Delta t+\sum_{j=1}^{p} \sum_{i=1}^{N} \int_{0}^{u^{i}\left(t_{j}\right)} I_{i j}(t) \mathrm{d} t+\int_{[0, T)_{\mathbb{T}}}\left(B u^{\sigma}(t), u^{\Delta}(t)\right) \Delta t \\
& -\frac{1}{2} \int_{[0, T)_{\mathbb{T}}}\left(A^{\sigma}(t) u^{\sigma}(t), u^{\sigma}(t)\right) \Delta t-\int_{[0, T)_{\mathbb{T}}} F\left(\sigma(t), u^{\sigma}(t)\right) \Delta t \\
\leq & -\delta\left\|u^{-}\right\|^{2}+\frac{1}{2} \int_{[0, T)_{\mathbb{T}}}\left|\left(u^{+}\right)^{\Delta}(t)\right|^{2} \Delta t+\int_{[0, T)_{\mathbb{T}}}\left(B\left(u^{+}\right)^{\sigma}(t),\left(u^{+}\right)^{\Delta}(t)\right) \Delta t \\
& -\frac{1}{2} \int_{[0, T)_{\mathbb{T}}}\left(A^{\sigma}(t)\left(u^{+}\right)^{\sigma}(t),\left(u^{+}\right)^{\sigma}(t)\right) \Delta t-\int_{[0, T)_{\mathbb{T}}} F\left(\sigma(t), u^{\sigma}(t)\right) \Delta t
\end{aligned}
$$




$$
\begin{aligned}
\leq & -\delta\left\|u^{-}\right\|^{2}+\frac{1}{2} \int_{[0, T)_{\mathbb{T}}}\left|\left(u^{+}\right)^{\Delta}(t)\right|^{2} \Delta t+\frac{\bar{b} N}{2} \int_{[0, T)_{\mathbb{T}}}\left|\left(u^{+}\right)^{\sigma}(t)\right|^{2} \Delta t \\
& +\frac{1}{4} \int_{[0, T)_{\mathbb{T}}}\left|\left(u^{+}\right)^{\Delta}(t)\right|^{2} \Delta t+\frac{C_{4}}{2} \int_{[0, T)_{\mathbb{T}}}\left|\left(u^{+}\right)^{\sigma}(t)\right|^{2} \Delta t-\int_{[0, T)_{\mathbb{T}}} F\left(\sigma(t), u^{\sigma}(t)\right) \Delta t \\
\leq & -\delta\left\|u^{-}\right\|^{2}+\frac{1}{2}\left(C_{4} C_{1}^{2}+\bar{b} N C_{1}^{2}+2\right)\left\|u^{+}\right\|^{2} \\
& -C_{9}^{2}\left(C_{4} C_{1}^{2}+\bar{b} N C_{1}^{2}+2+\delta\right)\|u\|_{L_{\Delta}^{2}}^{2}+C_{10} T+C_{11} \\
\leq & -\delta\left\|u^{-}\right\|^{2}+\left(C_{4} C_{1}^{2}+\bar{b} N C_{1}^{2}+2\right)\left\|u^{+}\right\|^{2} \\
& -\left(C_{4} C_{1}^{2}+\bar{b} N C_{1}^{2}+2+\delta\right)\|u\|^{2}+C_{10} T+C_{11} \\
= & -\delta\left\|u^{-}\right\|^{2}+\left(C_{4} C_{1}^{2}+\bar{b} N C_{1}^{2}+2\right)\left\|u^{+}\right\|^{2} \\
& -\left(C_{4} C_{1}^{2}+\bar{b} N C_{1}^{2}+2+\delta\right)\left\|u^{+}+u^{0}+u^{-}\right\|^{2}+C_{10} T+C_{11} \\
\leq & -\delta\left\|u^{-}\right\|^{2}+\left(C_{4} C_{1}^{2}+\bar{b} N C_{1}^{2}+2\right)\left\|u^{+}\right\|^{2}-\left(C_{4} C_{1}^{2}+\bar{b} N C_{1}^{2}+2+\delta\right)\left\|u^{+}\right\|^{2} \\
& -\delta\left\|u^{0}+u^{-}\right\|^{2}+C_{10} T+C_{11} \\
\leq & -\delta\left\|u^{-}\right\|^{2}+\left(C_{4} C_{1}^{2}+\bar{b} N C_{1}^{2}+2\right)\left\|u^{+}\right\|^{2} \\
& -\left(C_{4} C_{1}^{2}+\bar{b} N C_{1}^{2}+2+\delta\right)\left\|u^{+}\right\|^{2}-\delta\left\|u^{0}\right\|^{2}+C_{10} T+C_{11} \\
= & -\delta\|u\|^{2}+C_{10} T+C_{11},
\end{aligned}
$$

where $C_{11}=\max _{s \in\left[0, \rho_{6}\right]} a(s) \int_{[0, T)_{\mathbb{T}}} b^{\sigma}(t) \Delta t$. Hence, for every $n \in \mathbb{N}, \varphi(u) \rightarrow-\infty$ as $\|u\| \rightarrow$ $\infty$ and $X_{n}^{1} \oplus X^{2}$.

Thus, by Theorem 3.1, problem (1) has at least one nontrivial weak solution and one trivial weak solution.

Example 4.1 Let $T=3, N=4, t_{1}=1, t_{2}=2$. Consider the second order Hamiltonian system with impulsive effects

$$
\left\{\begin{array}{l}
\ddot{u}(t)+2 B \dot{u}(t)+A(t) u(t)+\nabla F(t, x)=0, \quad \text { a.e. } t \in[0,3] ; \\
u(0)-u(3)=\dot{u}(0)-\dot{u}(3)=0, \\
\Delta \dot{u}^{i}\left(t_{j}\right)=\dot{u}^{i}\left(t_{j}^{+}\right)-\dot{u}^{i}\left(t_{j}^{-}\right)=I_{i j}\left(u^{i}\left(t_{j}\right)\right), \quad i=1,2,3,4, j=1,2,
\end{array}\right.
$$

where $A(t)$ is the unit matrix,

$$
B=\left(\begin{array}{cccc}
0 & -1 & -2 & -3 \\
1 & 0 & -5 & -9 \\
2 & 5 & 0 & -2 \\
3 & 9 & 2 & 0
\end{array}\right)
$$

and

$$
F(t, x)=|x|^{4}, \quad \text { for all } x \in \mathbb{R}^{4} \text { and } t \in \mathbb{R},
$$




$$
I_{i j}(t)= \begin{cases}0, & t \geq 4 \\ -(t-4)^{5}, & 3 \leq t<4 \\ t-2, & 1<t<3 \\ -t^{5}, & |t| \leq 1 \\ t+2, & -3<t<-1 \\ -(t+4)^{5}, & -4<t \leq-3 \\ 0, & t \leq-4\end{cases}
$$

for all $i=1,2,3,4, j=1,2$. All conditions of Theorem 3.1 hold because of $\lambda=\eta=4$ and $\beta_{i j}=\gamma_{i j}=1, \xi_{i j}=5, \zeta_{i j}=4$, for all $i \in \Lambda_{1}, j \in \Lambda_{2}$. According to Theorem 4.1, problem (37) has at least one weak solution.

Theorem 4.2 Assume that $(\mathrm{A}),\left(\mathrm{F}_{1}\right),\left(\mathrm{F}_{3}\right),\left(\mathrm{F}_{5}\right),\left(\mathrm{F}_{6}\right),\left(\mathrm{F}_{7}\right)$, and the following conditions are satisfied.

(F $\left.\mathrm{F}_{8}\right) \lim \sup _{|x| \rightarrow 0} \frac{F(t, x)}{|x|^{2}} \leq 0$ uniformly for $\Delta$-a.e. $t \in[0, T]_{\mathbb{T}}$,

$\left(\mathrm{F}_{9}\right) F(t, x) \geq 0$, for all $x \in \mathbb{R}^{N}$ and $\Delta$-a.e. $t \in[0, T]_{\mathbb{T}}$.

Then problem (1) has at least one nontrivial weak solution.

Proof Set $E_{1}=H^{+}, E_{2}=H^{-} \oplus H^{0}$ and $E=H_{\Delta, T}^{1}$. Then $E$ is a real Hilbert space, $E=E_{1} \oplus E_{2}$, $E_{2}=E_{1}^{\perp}$, and $\operatorname{dim}\left(E_{2}\right)<+\infty$.

From the proof of Lemma 4.1, we know that $\varphi$ satisfies condition $(C)$.

On the other hand, for any small $\epsilon_{3}=\frac{3 \delta}{8 C_{1}^{2}}$, by $\left(\mathrm{F}_{8}\right)$ we know that there is a $\rho_{7}>0\left(\rho_{7}<\rho_{1}\right)$ such that

$$
F(t, x) \leq \epsilon_{3}|x|^{2}, \quad \text { for }|x|<\rho_{7}, \Delta \text {-a.e. } t \in[0, T]_{\mathbb{T}} .
$$

By $\left(\mathrm{F}_{7}\right)$, for $\epsilon_{4}=\frac{\delta}{8 p N C_{1}^{2}}$, there exists $\rho_{8}>0$ such that

$$
\left|I_{i j}(t)\right| \leq \epsilon_{4}|t|, \quad|t| \leq \rho_{8}, i \in \Lambda_{1}, j \in \Lambda_{2} .
$$

Let $\rho_{9}=\frac{1}{2} \min \left\{\rho_{7}, \rho_{8}\right\}$. For $u \in E_{1}$ with $\|u\| \leq r_{1} \triangleq \frac{\rho_{9}}{C_{1}}$, by (7), (9), (14), (38), and (39), we have

$$
\begin{aligned}
\varphi(u) & =q(u)+\sum_{j=1}^{p} \sum_{i=1}^{N} \int_{0}^{u^{i}\left(t_{j}\right)} I_{i j}(t) \mathrm{d} t-\int_{[0, T)_{\mathbb{T}}} F\left(\sigma(t), u^{\sigma}(t)\right) \Delta t \\
& \geq \delta\|u\|^{2}-\sum_{j=1}^{p} \sum_{i=1}^{N} \int_{0}^{\left|u^{i}\left(t_{j}\right)\right|}\left|I_{i j}(t)\right| \mathrm{d} t-\epsilon_{3} \int_{[0, T)_{\mathbb{T}}}\left|u^{\sigma}(t)\right|^{2} \Delta t \\
& \geq \delta\|u\|^{2}-\sum_{j=1}^{p} \sum_{i=1}^{N} \int_{0}^{\left|u^{i}\left(t_{j}\right)\right|} \epsilon_{4}|t| \mathrm{d} t-\epsilon_{3} \int_{[0, T)_{\mathbb{T}}}\left|u^{\sigma}(t)\right|^{2} \Delta t \\
& \geq \delta\|u\|^{2}-\epsilon_{4} \sum_{j=1}^{p} \sum_{i=1}^{N}\|u\|_{\infty}^{2}-\epsilon_{3} \int_{[0, T)_{\mathbb{T}}}\left|u^{\sigma}(t)\right|^{2} \Delta t \\
& \geq \delta\|u\|^{2}-\epsilon_{4} p N C_{1}^{2}\|u\|^{2}-\epsilon_{3} C_{1}^{2}\|u\|^{2}
\end{aligned}
$$




$$
\begin{aligned}
& \geq \delta\|u\|^{2}-\frac{\delta}{8}\|u\|^{2}-\frac{3 \delta}{8} \mid u \|^{2} \\
& =\frac{\delta}{2}\|u\|^{2} .
\end{aligned}
$$

Consequently,

$$
\varphi(u) \geq \frac{\delta \rho_{9}}{2} \triangleq \sigma>0, \quad \forall u \in E_{1} \text { with }\|u\|=\rho_{9}
$$

Moreover, we can prove that $J^{\prime}$ is compact as the same as the proof in [6]. It follows from (12), (40), and Lemma 3.2 that $\varphi$ satisfies the conditions $\left(\mathrm{I}_{5}\right),\left(\mathrm{I}_{6}\right)$, and $\left(\mathrm{I}_{7}\right)(\mathrm{i})$ with $S=\partial B_{\rho_{9}} \cap$ $E_{1}$ of Theorem 3.2.

Set $e \in E_{1} \cap \partial B_{1}, r_{3}>\rho_{9}, r_{4}>0, Q=\left\{s e: s \in\left(0, r_{3}\right)\right\} \oplus\left(B_{r_{4}} \cap E_{2}\right)$ and $\widetilde{E}=\operatorname{span}\{e\} \oplus E_{2}$. Then $S$ and $\partial Q$ link, where $B_{r_{4}}=\left\{u \in E:\|u\| \leq r_{4}\right\}$. Set

$$
Q_{1}=\left\{u \in E_{2}:\|u\| \leq r_{4}\right\}, \quad Q_{2}=\left\{r_{3} e+u: u \in E_{2}, \text { and }\|u\| \leq r_{4}\right\}
$$

and

$$
Q_{3}=\left\{s e+u: s \in\left[0, r_{3}\right], u \in E_{2} \text { and }\|u\|=r_{4}\right\} \text {. }
$$

Then $\partial Q=Q_{1} \cup Q_{2} \cup Q_{3}$.

$\operatorname{By}\left(F_{9}\right),(12),(13)$, and (32) we know $\left.\varphi\right|_{Q_{1}} \leq 0$. For each $r_{3} e+u \in Q_{2}$, one has $u=u^{0}+u^{-} \in$ $E_{2}$ and $\|u\| \leq r_{4}$. Hence there exists $C_{12}>0$ such that

$$
\left\|r_{3} e+u\right\|_{\infty} \leq C_{12}, \quad \forall r_{3} e+u \in Q_{2}
$$

By $\left(\mathrm{F}_{1}\right)$, for large $M>0$, there is $\rho_{10}>0$ such that

$$
F(t, x) \geq M|x|^{2}, \quad \forall|x| \geq \rho_{10}, \Delta \text {-a.e. } t \in[0, T]_{\mathbb{T}} .
$$

By the equivalence of a finite dimensional space, $\left(\mathrm{F}_{9}\right),(41)$, and (42), there exists $C_{13}>0$ such that

$$
\begin{aligned}
\int_{[0, T)_{\mathbb{T}}} F\left(\sigma(t), r_{3} e^{\sigma}(t)+u^{\sigma}(t)\right) \Delta t & \geq M \int_{[0, T)_{\mathbb{T}}}\left|r_{3} e^{\sigma}(t)+u^{\sigma}(t)\right|^{2} \Delta t-M C_{12}^{2} T \\
& \geq M C_{13}\left\|r_{3} e+u\right\|^{2}-M C_{12}^{2} T \\
& =M C_{13}\left(r_{3}^{2}+\|u\|^{2}\right)-M C_{12}^{2} T .
\end{aligned}
$$

Thus, by (32) and (43) we have

$$
\begin{aligned}
\varphi\left(r_{3} e+u\right)= & \frac{r_{3}^{2}}{2}\langle(I-K) e, e\rangle+\frac{1}{2}\langle(I-K) u, u\rangle+\phi\left(r_{3} e+u\right) \\
& -\int_{[0, T)_{\mathbb{T}}} F\left(\sigma(t), r_{3} e^{\sigma}(t)+u^{\sigma}(t)\right) \Delta t \\
\leq & \frac{r_{3}^{2}}{2}\|I-K\|-\delta\left\|u^{-}\right\|^{2}-M C_{13}\left(r_{3}^{2}+\|u\|^{2}\right)+M C_{12}^{2} T
\end{aligned}
$$




$$
\begin{aligned}
& \leq-\left(M C_{13}-\frac{1}{2}\|I-K\|\right) r_{3}^{2}+M C_{12}^{2} T \\
& \leq 0
\end{aligned}
$$

for large $M>0$ and $r_{3}>\rho_{9}$.

Moreover, for each $s e+u \in Q_{3}$, one has $s \in\left[0, r_{3}\right], u \in E_{2}$, and $\|u\|=r_{4}$. Hence there exists $C_{14}>0$ such that

$$
\|s e+u\|_{\infty} \leq C_{14}, \quad \forall s e+u \in Q_{3} .
$$

By the equivalence of a finite dimensional space, $\left(\mathrm{F}_{9}\right),(42)$, and (44), we have

$$
\begin{aligned}
\int_{[0, T)_{\mathbb{T}}} F\left(\sigma(t), s e^{\sigma}(t)+u^{\sigma}(t)\right) \Delta t & \geq M \int_{[0, T)_{\mathbb{T}}}\left|s e^{\sigma}(t)+u^{\sigma}(t)\right|^{2} \Delta t-M C_{14}^{2} T \\
& \geq M C_{13}\|s e+u\|^{2}-M C_{14}^{2} T \\
& =M C_{13}\left(s^{2}+\|u\|^{2}\right)-M C_{14}^{2} T \\
& =M C_{13}\left(s^{2}+r_{4}^{2}\right)-M C_{14}^{2} T .
\end{aligned}
$$

Thus, by (32) and (45) we have

$$
\begin{aligned}
\varphi(s e+u)= & \frac{s^{2}}{2}\langle(I-K) e, e\rangle+\frac{1}{2}\langle(I-K) u, u\rangle+\phi(s e+u) \\
& -\int_{[0, T)_{\mathbb{T}}} F\left(\sigma(t), s e^{\sigma}(t)+u^{\sigma}(t)\right) \Delta t \\
\leq & \frac{s^{2}}{2}\|I-K\|-\delta\left\|u^{-}\right\|^{2}-M C_{13}\left(s^{2}+r_{4}^{2}\right)+M C_{14}^{2} T \\
\leq & -\left(M C_{13}-\frac{1}{2}\|I-K\|\right) s^{2}-M C_{13} r_{4}^{2}+M C_{12}^{2} T \\
\leq & 0
\end{aligned}
$$

for large $M>0$ and $r_{4}>0$.

Summing up the above, $\varphi$ satisfies all conditions of Theorem 3.2. Hence $\varphi$ possesses a critical value $c \geq \sigma>0$, and hence problem (1) has at least one non-trivial weak solution. The proof is complete.

Remark 4.1 There are a number of functions satisfying $(A),\left(F_{1}\right),\left(F_{3}\right),\left(F_{8}\right)$, and $\left(F_{9}\right)$, for example, $F(t, x)=e^{2 t}|x|^{4}$.

Next, we give two multiplicity results.

Theorem 4.3 Assume that (A), $\left(\mathrm{F}_{1}\right),\left(\mathrm{F}_{3}\right),\left(\mathrm{F}_{5}\right),\left(\mathrm{F}_{7}\right),\left(\mathrm{F}_{8}\right)$, and the following conditions are satisfied.

$\left(\mathrm{F}_{10}\right) I_{i j}\left(i \in \Lambda_{1}, j \in \Lambda_{2}\right)$ are odd.

$\left(\mathrm{F}_{11}\right) \quad F(t, x)$ is even in $x$ and $F(t, 0)=0$.

Then problem (1) has an unbounded sequence of weak solutions. 
Proof Set $W=H^{+}, V=H^{-} \oplus H^{0}$ and $E=H_{\Delta, T}^{1}$. Then $E=V \oplus W, \operatorname{dim} V<+\infty$, and $\varphi \in$ $C^{1}(E, R)$. From the proof of Lemma 4.1, we know that $\varphi$ satisfies the (C) condition. From the proof of Theorem 4.2, we know that there exist a $\rho_{9}>0$ and $\sigma>0$ such that

$$
\varphi(u) \geq \sigma, \quad \forall u \in W \text { with }\|u\|=\rho_{9} .
$$

For each finite dimensional subspace $\widetilde{E} \subset E$, by the equivalence of a finite dimensional space, there is a constant $C_{15}>0$ such that

$$
\int_{[0, T)_{\mathbb{T}}}\left|u^{\sigma}(t)\right|^{2} \Delta t \geq C_{15}\|u\|^{2}, \quad \forall u \in \widetilde{E}
$$

Set constant $M=\left(C_{4} C_{1}^{2}+\bar{b} N C_{1}^{2}+2\right)$. Then, by $\left(\mathrm{F}_{1}\right)$, there is $\rho_{11}>0$ such that

$$
F(t, x) \geq M|x|^{2}, \quad \forall|x| \geq \rho_{11} \text { and } \Delta \text {-a.e. } t \in[0, T] .
$$

By (A) and (47), one has

$$
F(t, x) \geq M|x|^{2}-M \rho_{11}^{2}-C_{16} b(t), \quad \forall x \in R^{n} \text { and } \Delta \text {-a.e. } t \in[0, T]
$$

where $C_{16}=\max _{s \in\left[0, \rho_{11}\right]} a(s)$. Hence, combining (9), (19), (20), (21), (46), and (48), for each $u \in \widetilde{E}$, one has

$$
\begin{aligned}
\varphi(u)= & \frac{1}{2} \int_{[0, T)_{\mathbb{T}}}\left|u^{\Delta}(t)\right|^{2} \Delta t+\sum_{j=1}^{p} \sum_{i=1}^{N} \int_{0}^{u^{i}\left(t_{j}\right)} I_{i j}(t) \mathrm{d} t+\int_{[0, T)_{\mathbb{T}}}\left(B u^{\sigma}(t), u^{\Delta}(t)\right) \Delta t \\
& -\frac{1}{2} \int_{[0, T)_{\mathbb{T}}}\left(A^{\sigma}(t) u^{\sigma}(t), u^{\sigma}(t)\right) \Delta t-\int_{[0, T)_{\mathbb{T}}} F\left(\sigma(t), u^{\sigma}(t)\right) \Delta t \\
\leq & \frac{1}{2} \int_{[0, T)_{\mathbb{T}}}\left|u^{\Delta}(t)\right|^{2} \Delta t+\frac{C_{4}}{2} \int_{[0, T)_{\mathbb{T}}}\left|u^{\sigma}(t)\right|^{2} \Delta t+\bar{\beta} p N C_{1}\|u\| \\
& +\bar{\gamma} C_{1}^{\xi_{i j}+1} \sum_{j=1}^{p} \sum_{i=1}^{N}\|u\|^{\xi_{i j}+1}+\frac{\bar{b} N}{2} \int_{[0, T)_{\mathbb{T}}}\left|u^{\sigma}(t)\right|^{2} \Delta t+\frac{1}{4} \int_{[0, T)_{\mathbb{T}}}\left|u^{\Delta}(t)\right|^{2} \Delta t \\
& -M \int_{[0, T)_{\mathbb{T}}}\left|u^{\sigma}(t)\right|^{2} \Delta t+M \rho_{11}^{2} T+C_{16} \int_{[0, T)_{\mathbb{T}}} b^{\sigma}(t) \Delta t \\
\leq & \frac{1}{2} \int_{[0, T)_{\mathbb{T}}}\left|u^{\Delta}(t)\right|^{2} \Delta t+\frac{C_{4}}{2} \int_{[0, T)_{\mathbb{T}}}\left|u^{\sigma}(t)\right|^{2} \Delta t+\bar{\beta} p N C_{1}\|u\| \\
& +\bar{\gamma} C_{1}^{\xi_{i j}+1} \sum_{j=1}^{p} \sum_{i=1}^{N}\|u\|^{\xi_{i j}+1} \\
& \bar{b} N \int_{[0, T)_{\mathbb{T}}}\left|u^{\sigma}(t)\right|^{2} \Delta t+\frac{1}{4} \int_{[0, T)_{\mathbb{T}}}\left|u^{\Delta}(t)\right|^{2} \Delta t-M C_{15}\|u\|^{2}+M \rho_{11}^{2} T+C_{17} \\
\leq & \frac{1}{2}\left(C_{4} C_{1}^{2}+\bar{b} N C_{1}^{2}+2-2 M\right)\|u\|^{2}+\bar{\beta} p N C_{1}\|u\| \\
& +\bar{\gamma} C_{1} \sum_{j=1}^{p} \sum_{i=1}^{N}\|u\|^{\xi_{i j}+1}+M \rho_{11}^{2} T+C_{17}, \\
&
\end{aligned}
$$


where $C_{17}=C_{16} \int_{[0, T)_{\mathbb{T}}} b^{\sigma}(t) \Delta t$, and hence

$$
\varphi(u) \rightarrow-\infty \quad \text { as } u \in \widetilde{E} \text { and }\|u\| \rightarrow \infty .
$$

This implies that there is an $R=R_{(\widetilde{E})}>0$ such that $\varphi \leq 0$ on $\widetilde{E} \backslash B_{R}$.

Moreover, by $\left(\mathrm{F}_{10}\right)$ and $\left(\mathrm{F}_{11}\right)$, we know that $\varphi$ is even and $\varphi(0)=0$. In view of Theorem 3.3, $\varphi$ has a sequence of critical point $\left\{u_{n}\right\} \subset E$ such that $\left|\varphi\left(u_{n}\right)\right| \rightarrow \infty$. If $\left\{u_{n}\right\}$ is bounded in $E$, then by the definition of $\varphi$, one knows that $\left\{\left|\varphi\left(u_{n}\right)\right|\right\}$ is also bounded, a contradiction. Hence $\left\{u_{n}\right\}$ is unbounded in $E$. The proof is completed.

Remark 4.2 There are a number of functions satisfying $(A),\left(F_{1}\right),\left(F_{3}\right),\left(F_{8}\right)$, and $\left(F_{11}\right)$, for example, $F(t, x)=\left(|x|^{6}\right)$. Moreover, there are a number of functions satisfying $\left(\mathrm{F}_{5}\right),\left(\mathrm{F}_{7}\right)$, $\left(\mathrm{F}_{10}\right)$, and not satisfying $\left(\mathrm{F}_{6}\right)$, for example,

$$
I_{i j}(t)= \begin{cases}0, & t \geq 4 \\ (t-4)^{3}, & 3 \leq t<4 \\ -t+2, & 1<t<3 \\ t^{3}, & |t| \leq 1 \\ -t-2, & -3<t<-1 \\ (t+4)^{3}, & -4<t \leq-3 \\ 0, & t \leq-4\end{cases}
$$

for all $i=1,2,3,4, j=1,2$.

Remark 4.3 In Theorem 4.3 if we delete the condition ' $F(t, 0)=0$ ', we have the following theorem.

Theorem 4.4 Assume that $(\mathrm{A}),\left(\mathrm{F}_{1}\right),\left(\mathrm{F}_{3}\right),\left(\mathrm{F}_{5}\right),\left(\mathrm{F}_{7}\right),\left(\mathrm{F}_{8}\right),\left(\mathrm{F}_{10}\right)$, and the following condition is satisfied.

$\left(\mathrm{F}_{12}\right) F(t, x)$ is even in $x$.

Then problem (1) has an infinite sequence of distinct weak solutions.

Proof Set $Y=H^{+}, X=H^{-} \oplus H^{0}$ and $E=H_{\Delta, T}^{1}$ in Theorem 3.4. Then, from the proof of Lemma 4.1 and Theorem 4.3, we know that $E=X \oplus Y, \operatorname{dim}(X)<+\infty, \varphi$ is even, $\varphi \in C^{1}(E, R)$ satisfies condition $(C)$ and there are constants $\rho_{9}, \sigma>0$ such that $\left.\varphi\right|_{\partial B_{\rho 9} \cap Y} \geq \sigma$ and inf $\varphi\left(B_{\rho_{9}} \cap Y\right) \geq 0$, where $\partial B_{\rho_{9}}=\left\{u \in E:\|u\|=\rho_{9}\right\}$.

For each finite dimensional subspace $\widetilde{E} \subset E$, by (49), we know that

$$
\varphi(u) \rightarrow-\infty \quad \text { as } u \in \widetilde{E} \text { and }\|u\| \rightarrow \infty .
$$

Consequently, for each finite dimensional subspace $Y_{0} \subset Y$, the condition $\left(\Phi_{2}\right)$ holds. Moreover, by $\operatorname{dim}(X)<+\infty$ and $\varphi \in C^{1}(E, R)$, we know that $\left(\Phi_{0}\right)$ holds, too. Therefore, the conclusion follows from Theorem 3.4.

Remark 4.4 Even if in the case that $I_{i j} \equiv 0, i \in \Lambda_{1}, j \in \Lambda_{2}, \mathbb{T}=\mathbb{R}$, Theorem 4.3 and Theorem 4.4 are new too. 


\section{Competing interests}

The authors declare that they have no competing interests.

\section{Authors' contributions}

All authors wrote, read and approved the final manuscript.

\section{Acknowledgements}

This work is supported by the National Natural Sciences Foundation of People's Republic of China under Grant 11361072,11326101 and 20125301120007 and the Natural Sciences Foundation of Yunnan Province under Grant 2012 FB111.

Received: 8 December 2014 Accepted: 17 February 2015 Published online: 04 March 2015

\section{References}

1. Mawhin, J, Willem, M: Critical Point Theory and Hamiltonian Systems. Springer, Berlin (1989)

2. Berger, MS, Schechter, M: On the solvability of semilinear gradient operator equations. Adv. Math. 25, 97-132 (1977)

3. Long, YM: Nonlinear oscillations for classical Hamiltonian systems with bi-even subquadratic potentials. Nonlinear Anal. 24, 1665-1671 (1995)

4. He, XM, Wu, X: Periodic solutions for a class of nonautonomous second order Hamiltonian systems. J. Math. Anal. Appl. 341, 1354-1364 (2008)

5. Meng, FJ, Zhang, FB: Periodic solutions for some second order systems. Nonlinear Anal. 68, 3388-3396 (2008)

6. Wu, X, Chen, SX, Teng, KM: On variational methods for a class of damped vibration problems. Nonlinear Anal. 68 , $1432-1441(2008)$

7. Li, X, Wu, X, Wu, K: On a class of damped vibration problems with super-quadratic potentials. Nonlinear Anal. 72 , 135-142 (2010)

8. Zhou, JW, Li, YK: Variational approach to a class of second order Hamiltonian systems on time scales. Acta Appl. Math. 117, 47-69 (2012)

9. Su, YH, Feng, Z: A non-autonomous Hamiltonian systems on time scales. Nonlinear Anal. 75, 4126-4136 (2012)

10. Yang, L, Liao, YZ, Li, YK: Existence and exponential stability of periodic solutions for a class of Hamiltonian systems on time scales. Adv. Differ. Equ. 2013, Article ID 180 (2013)

11. Song, WJ, Gao, WJ: Existence of solutions for nonlocal p-Laplacian thermistor problems on time scales. Bound. Value Probl. 2013, Article ID 1 (2013)

12. Tokmak, F, Karaca, LY: Existence of symmetric positive solutions for a multipoint boundary value problem with sign-changing nonlinearity on time scales. Bound. Value Probl. 2013, Article ID 52 (2013)

13. Li, YK, Yang, L, Sun, LJ: Existence and exponential stability of an equilibrium point for fuzzy BAM neural networks with time-varying delays in leakage terms on time scales. Adv. Differ. Equ. 2013, Article ID 218 (2013)

14. Su, YH, Feng, Z: Homoclinic orbits and periodic solutions for a class of Hamiltonian systems on time scales. J. Math. Anal. Appl. 411, 37-62 (2014)

15. Friesl, M, Slavik, A, Stehlik, P: Discrete-space partial dynamic equations on time scales and applications to stochastic processes. Appl. Math. Lett. 37, 86-90 (2014)

16. Hilger, S: Analysis on measure chains - a unified approach to continuous and discrete calculus. Results Math. 18 18-56 (1990)

17. Agarwal, RP, Otero-Espinar, V, Perera, K, Vivero, DR: Basic properties of Sobolev's spaces on time scales. Adv. Differ. Equ. 2006, Article ID 38121 (2006)

18. Aulbach, B, Hilger, S: Linear dynamic processes with inhomogeneous time scale. In: Nonlinear Dynamics and Quantum Dynamical Systems (Gaussig, 1990). Math. Res., vol. 59, pp. 9-20. Akademie Verlag, Berlin (1990)

19. Otero-Espinar, V, Pernas-Castaño, T: Variational approach to second-order impulsive dynamic equations on time scales. Bound. Value Probl. 2013, Article ID 119 (2013)

20. Zhou, JW, Li, YK: Sobolev's spaces on time scales and its applications to a class of second order Hamiltonian systems on time scales. Nonlinear Anal. 73, 1375-1388 (2010)

21. Bartolo, P, Benci, V: Abstract critical point theorems and applications to some nonlinear problems with 'strong' resonance at infinity. Nonlinear Anal. 7, 981-1012 (1983)

22. Luan, SX, Mao, AM: Periodic solutions for a class of non-autonomous Hamiltonian systems. Nonlinear Anal. 61 , 1413-1426 (2005)

23. Rabinowitz, PH: Minimax Methods in Critical Point Theory with Application to Differential Equations. CBMS Regional Conf. Ser. in Math., vol. 65. Am. Math. Soc., Providence (1986)

24. Bartsch, T, Ding, YH: Deformation theorems on non-metrizable vector spaces and applications to critical point theory. Math. Nachr. 279(12), 1267-1288 (2006) 